\title{
The effects of cognitive-motor training interventions on executive functions in older people: a systematic review and meta-analysis
}

Bettina Wollesen ${ }^{1,2^{*}}$ (D), Alicia Wildbredt ${ }^{1}$, Kimberley S. van Schooten ${ }^{3,4}$, Mei Ling Lim ${ }^{3,4}$ and Kim Delbaere 3,4

\begin{abstract}
Background: Ageing is associated with physical and cognitive decline, affecting independence and quality of life in older people. Recent studies show that in particular executive functions are important for daily-life function and mobility. This systematic review investigated the effectiveness of cognitive-motor training including exergaming on executive function (EF, set-shifting, working memory, inhibitory control) in healthy older people.

Methods: An electronic database search for randomised controlled trials (RCT), controlled clinical trials (CCT) and parallel group trials was performed using MEDLINE, EMBASE, and PsychINFO following PRISMA guidelines. Inclusion criteria were: (1) community-dwelling participants > 60 years without a medical condition or medical treatment, (2) reporting at least one cognitive-motor intervention while standing or walking, (3) use of dual-task interventions using traditional methods or modern technology to deliver a cognitive-motor task, (4) inclusion of at least one cognitive outcome. The PEDro scale was used for quality assessment.

Results: A total of 1557 studies were retrieved, of which 25 studies were included in this review. Eleven studies used a technology-based dual-task intervention, while 14 trials conducted a general cognitive-motor training. The age range of the cohort was 69 to 87 years. The interventions demonstrated positive effects on global cognitive function [mean difference $0.6,95 \% \mathrm{Cl} 0.29-0.90$ ] and inhibitory control [mean difference $0.61,95 \% \mathrm{Cl} 0.28-0.94$ ]. Effects were heterogeneous ( $\left.\right|^{2}$ range: $\left.60-95\right)$ and did not remain after a sensitivity analysis. Processing speed and dual-task costs also improved, but meta-analysis was not possible.

Conclusion: Cognitive-motor and technology-based interventions had a positive impact on some cognitive functions. Dual-task interventions led to improvements of domains related global cognitive functions and inhibitory control. Likewise, technology-based exergame interventions improved functions related to processing speed, attentional and inhibitory control. Training interventions with a certain level of exercise load such as progression in difficulty and task specificity were more effective to gain task-related adaptations on cognitive functions.
\end{abstract}

Keywords: Aged ${ }_{1}$, Dual-task ${ }_{2}$ Exergame 3 , Motor-cognitive intervention ${ }_{4}$, Cognitive function $_{5}$

\footnotetext{
* Correspondence: Bettina.wollesen@uni-hamburg.de

'Department of Human Movement Science, University of Hamburg,

Hamburg, Germany

${ }^{2}$ Biological Psychology and Neuroergonomics, Technical University of Berlin,

Fasanenstr. 1, D-10623 Berlin, Germany

Full list of author information is available at the end of the article
}

C C The Author(s). 2020 Open Access This article is licensed under a Creative Commons Attribution 4.0 International License, which permits use, sharing, adaptation, distribution and reproduction in any medium or format, as long as you give appropriate credit to the original author(s) and the source, provide a link to the Creative Commons licence, and indicate if changes were made. The images or other third party material in this article are included in the article's Creative Commons licence, unless indicated otherwise in a credit line to the material. If material is not included in the article's Creative Commons licence and your intended use is not permitted by statutory regulation or exceeds the permitted use, you will need to obtain permission directly from the copyright holder. To view a copy of this licence, visit http://creativecommons.org/licenses/by/4.0/. The Creative Commons Public Domain Dedication waiver (http://creativecommons.org/publicdomain/zero/1.0/) applies to the data made available in this article, unless otherwise stated in a credit line to the data. 


\section{Introduction}

Ageing is associated with changes to the locomotor system and reduced efficiency of cognitive processing [1]. Most daily activities require the simultaneous execution of cognitive and motor tasks [2]. Limited resources, reduced efficiency and increased interference between tasks often lead to deterioration of motor and/or cognitive performance with ageing [3, 4]. Reduced performance while performing a simultaneous task, often referred to as cognitive-motor interference, represents a major risk factor of falls in older people [5-8]. Therefore, adequate physical and cognitive resources with the ability to use these resources simultaneously seems key in order for older people to maintain an independent lifestyle.

Executive functions (EF) are the main cognitive abilities necessary to interact with the environment in daily activities [9]. Executive functions include a wide range of higher-order cognitive functions. A recent review by Diamond [10] identified three major domains of EF that are of particular relevance to walking and daily life activities [11-13]: (i) inhibitory control, which includes the ability to stay focused despite distraction and inhibit pre-potent but inappropriate responses; (ii) working memory, which refers to the ability to hold and manipulate information, prioritise and plan actions, and (iii) cognitive flexibility, which refers to one's ability to adjust and change attention, as well as set-shifting and task-switching (cf. Textbox 1).

\section{Textbox 1- Cognition and executive function taxonomy}

The term "cognition" is a broad expression of mental domains related to brain processes connected to assimilating and understanding external stimulation [14] and "the ability to hold, process and manipulate information in the mind" ([15], p.5).

One major important set of cognitive processing is described by the term "executive function" (EF), which is also referred to as cognitive control [10]. According to the definition of Diamond [10], EF is an umbrella term for a collection of mental processes regarding the ability to concentrate, focus and adequately react to external stimuli.

Based on this it is of general agreement that EFs are subdivided into three core elements inhibition, working memory and cognitive flexibility [10].

1. Inhibition describes the ability to control attentional resources, e.g.

connected to behaviour, thoughts or emotions. Inhibition enables an

individual to selectively focus on an external stimulus to be

processed, while suppressing other stimuli.

- Inhibitory control is often measured by testing one's selective attention which requires the ability to correctly discriminate two incongruent stimuli, e.g. the Stroop, the Flanker Test or tasks involving a go/no-go instruction.

2. Working memory (WM) involves holding and manipulating information in mind, e.g. using stored information to solve an ongoing problem.

Linked to a variety of neuronal subsystems, WM enables the analysis and

clustering of information while selectively focusing on information stored

in mind. Testing WM often requires reordering stored information, e.g.

repeat a selection of numbers in another order, which is for example used in complex span-backwards tasks, e.g. the n-back task.

- Verbal fluency tasks require the ability to hold information in mind, while performing a mental process, e.g. the spontaneous production of words.

- Arithmetic tasks refer to tasks solving mathematical problems, e.g. counting backwards or multiplying.

3. Cognitive flexibility describes the ability to change perspectives according to external demands and an adequate reaction. Flexibility is often investigated via task switching (set-shifting) tasks. This requires the ability to randomly shift between various required stimulus-respond set, e.g. during the Wisconsin Sorting Card test [15].

Other basic cognitive functions regarding reaction time and speed of cognitive processing [16]:

1. Processing speed is referred to as the simple reaction time between an external stimulus and a behavioural response occurrence, which can be tested by the finger-tapping speed as a reaction of a visual stimulus.

2. Visuospatial abilities are connected to the processing and memory of visual as well as spatial stimuli. Common test for visuospatial planning abilities are the Clock-Drawing-Test or the Spatial Span test.

Previous research has shown that it is possible to improve cognitive function in older people, using physical and mental training, individually or combined. Combining physical and mental training is often referred to as dual-task training (DT) $[8,17,18]$. Dual-tasking describes the combination of a motor and a cognitive task; e.g. walking while counting backwards, conducting a verbal fluency task (e.g. naming animals), attentional control (e.g. reaction on signs) and processing speed [8]. Generally, simultaneous demands can lead to a reduced performance in one of the two tasks. The change in performance under dual-task compared to single-task conditions is called a dual-task cost [7], otherwise known as cognitive-motor interference [19]. In a recent review, Tait and colleagues [18] demonstrated that training motor and cognitive tasks simultaneously (dual-task training) showed significant improvements in cognitive functions, i.e. global cognition and EF (inhibition, working memory and cognitive flexibility), compared to sequential physical and mental training.

Recent studies have repeatedly shown that the ageing brain and body remain plastic and that older people's performance can be improved through systematic motor or cognitive training [20-22]. However, improvements in neurophysiological correlates differ with the type of the training tasks [21]. Thus, through physical training, cognitive resources can be applied more effectively. Moreover, different types of dual- or multi-task training can positively influence cognitive performance of older people $[8,21]$. While there are positive results, more work is required to uncover which types of dual-task training are most effective. Wollesen and VoelckerRehage [8] subdivided dual-task training into categories based on the training and assessment method, differentiating general and specific dual-task training. General dual-tasking (GDT) describes an intervention which uses a variety of dual-task exercises with the idea that this intervention might improve general dual-task performance. Specific dual-tasking (SDT) requires the participant to specifically conduct the same tasks as in the training condition. In line with these classifications, a General Dual-Task (GDT) program describes an 
intervention which uses different unspecific training tasks in comparison to the assessment tasks; while a Specific Dual-Task (SDT) program consists of training tasks that address a comparable cognitive dimension as the assessment task (cf. Textbox 2).

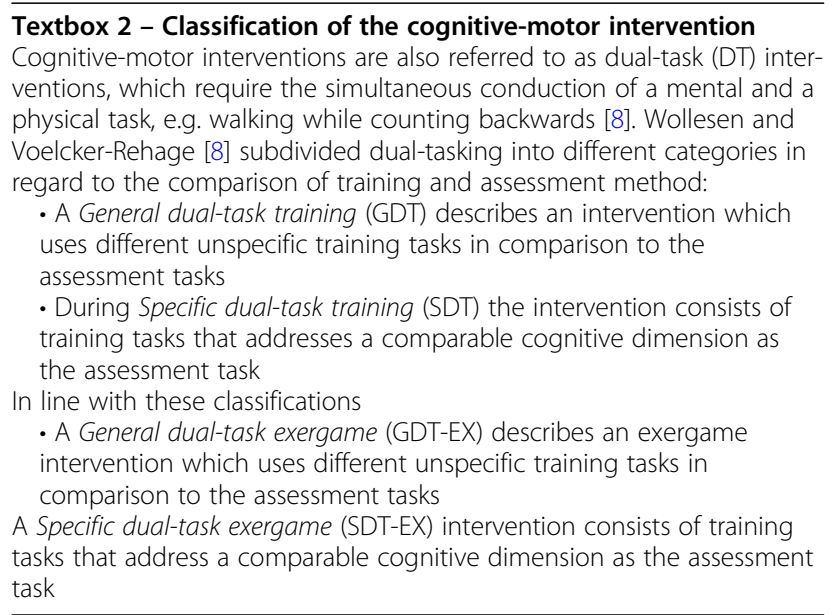

Dual-task training programs have traditionally been delivered face-to-face in group settings or one-on-one. Positive effects on cognitive-motor performance can be observed if the training has a minimum duration of twelve sessions or $330 \mathrm{~min}$ as well as an advancement in task difficulty and progression [8]. Due to rapidly changing technologies, it has become more popular to utilise exercised-based computer games, also known as exergames, for dual-task training. If well-designed, exergames can add a high cognitive demand to physical training, possibly even making use of a virtual environment [19]. Exergaming might also have the benefit of being implemented as an unsupervised, home-based program, which can potentially reduce costs for users.

The purpose of this review was twofold: (i) to summarise existing studies using cognitive-motor interventions and their effects on global cognitive functioning as well as the three dimensions of EF (inhibitory control, working memory and cognitive flexibility) in older people aged 60 years and above, and (ii) to identify the common characteristics of effective interventions in terms of duration, frequency and task progression. We hypothesise that EF can be improved by a specific DT intervention including at least a duration of $330 \mathrm{~min}$ or twelve sessions and a progression of task difficulty and task complexity.

\section{Methods}

The protocol for this review was prospectively registered with the International Prospective Register of Systematic Reviews (PROSPERO) with the registration number CRD 42018111083.

\section{Search strategy and selection criteria}

A systematic electronic database search for relevant articles was performed using MEDLINE (1966 to 2019, week 40), EMBASE (1966 to 2019, week 40) and PsychINFO (1980 to 2019, week 40). The literature search included keywords related to older people, cognitive-motor as well as technology-based or exergaming interventions, and a cognitive outcome (cf. Table 1 for more details; a detailed list of the key terms is added as an Appendix). The process also included a manual search of the reference lists of relevant articles to identify articles that did not show up in the search strategy results. The search within the databases was limited to articles published in English. Two reviewers (AW and $\mathrm{BW}$ ) independently reviewed the articles by title and abstract to identify all potentially eligible articles following the PRISMA methodology [23]. The process integrated the screening of the articles by title and abstract. All relevant articles regarding title and abstract were integrated into the full text screening. If the screening of the abstract did not clarify the inclusion or exclusion criteria, the full text was extracted. Afterwards, the two reviewers independently assessed full version copies of all potentially eligible articles to determine the ones to be included. Any disagreement on inclusion was resolved by discussion and through consensus by the larger project team (including KvS, ML and KD).

The study selection was limited to randomised controlled trials, control group designs trials and parallel group trials. Study protocols, abstracts or conference abstracts were excluded due to limited information. Articles were included if they met the methodological inclusion and exclusion criteria.

The target population included community-dwelling participants with a minimum age of 60 and a mean age above 65 without a medical condition or recent medical treatment. This included participants who lived independently in private homes, in independent-living units or retirement villages. We included participants with no cognitive deficits or with a diagnosis of mild cognitive impairment only. Therefore, we agreed to exclude studies including participants with pre-intervention scores below 25 on the MMSE or equivalent on the Montreal Cognitive Assessment (MoCA) score. Trials in which participants were selected based on a medical condition (e.g. stroke, Parkinson disease, multiple sclerosis, schizophrenia or dementia) and trials including only participants who received a recent medical treatment (e.g. surgery or medication) were excluded.

Trials with interventions using at least one cognitivemotor exercise while standing or walking were included. The training could be delivered using traditional dualtasking methods or modern technology. Dual-tasking or cognitive-motor interventions required simultaneous 
cognitive and motor exercise. Additionally, interventions using technologies could use consoles, virtual reality systems or bespoke software. Trials presenting on seated training programs were excluded. We extracted whether the motor task was conducted while standing or walking due to the different levels of complexity of the motor tasks. When the intervention required the participant to work with a visual feedback or biofeedback as part of the technology-based game, this was considered a cognitive load.

Finally, each study had at least one cognitive outcome, evaluated as a primary or secondary outcome. These cognitive domains included general cognition, general executive function, set-shifting, inhibitory control, working memory, visuospatial planning, verbal fluency, attentional control, processing speed and dual-task costs.

\section{Data extraction and quality assessment}

Eligible articles were screened independently by two review authors (AW, BW). Data on sample characteristics, type of a traditional motor-cognitive or technological exergaming intervention as well the frequency and duration of the intervention was extracted. Table 1 provides an overview of all included articles including the authors, year of publication, included participants, study aims, intervention, cognitive and motor task measurement, and training duration.

To assess risk of bias and quality, we used the PEDro scale [24]. Articles scoring 6-10 points on the PEDro quality assessment were considered to be of high quality, articles with a score of 4-5 of fair quality and articles with a score of $0-3$ of poor quality (cf. Table 2).

\section{Statistical analysis of the meta-analysis}

We extracted data of the intervention and control group for each of the outcome variables of interest (cf. Textbox 1) as a difference in means (MD). Most of the studies reported means and Standard Deviations (SD) permitting effect size estimation, otherwise, these were derived from summary statistics reported in the articles, such as $t$-values or $p$-values. The cognitive data from individual studies were then pooled in meta-analyses to estimate the overall effect of the training intervention on cognition. Studies were grouped by cognitive task domain and individual meta-analyses were conducted for each EF outcome. As the studies used different control groups (e.g. active controls with another intervention, for example a single-task motor intervention or an education program or inactive controls with normal daily activities cf. Table 3), the meta-analysis was conducted with a subgroup analysis regarding active and inactive controls. A random-effects model with a generic inverse variance method was used, which gives more weight to studies with less variance in the pooled analyses. Results are presented as effect size with $95 \%$ confidence interval (CI) and respective values for null hypothesis tests (e.g. cognitive-motor training has no effect on EF). Heterogeneity between studies was investigated by calculating the Q-value and $\mathrm{I}^{2}$ statistic, which quantified the proportion variation that is due to heterogeneity rather than chance. Quantitative syntheses and meta-analyses were produced using Review Manager 5 Software (RevMan 5).

\section{Results}

\section{Classification of included studies}

The initial search generated 2593 articles of which 705 were duplicates (Fig. 1). For the final review, 25 articles were identified eligible and integrated into further assessment.

\section{Cohort characteristics}

Studies included samples of 13 to 134 participants (median 36). The mean age of participants ranged from 66.8 to 86.0 years (cf. Table 1). Both genders were included in 18 of the trials, generally with a higher percentage of female participants. Seven studies did not provide any information on the gender of their participants [25-31].

\section{Quality assessment and bias}

Based on the quality assessment, 17 articles were considered to be of high quality, five of fair quality and only three articles of low quality. Eighteen of the included articles were RCTs, of which 15 were of high and three of fair quality. Two of the seven controlled trials were rated of high quality, two of fair quality and three of low quality. In general, most studies reported the in/exclusion criteria (92\%), comparable baseline characteristic for the control and intervention groups on the most important prognostic indicators (80\%), and group comparisons (80\%). All studies provided a measurement of variability for at least one key outcome. Only three studies (12\%) blinded their participants. Four studies (16\%) reported blinding of the therapists who administered the exercise intervention, and only three studies trials (12\%) reported blinding of all assessors for at least one key outcome (cf. Table 2).

\section{Intervention characteristics}

The intervention characteristics, including the type of intervention, a description of the motor and cognitive components, frequency and dose are outlined in Table 3.

The cognitive load, which was combined with the motor exercise, showed different levels of cognitive demand. Exergaming situations differed between commercially available computer games to laboratorybased training forms. In many cases the digital game required players to interact with objects on a screen in the 
Table 1 Characteristics of included studies

\begin{tabular}{|c|c|c|c|c|c|c|}
\hline $\begin{array}{l}\text { Eligible author } \\
\text { and year }\end{array}$ & $\begin{array}{l}\text { Participants } \\
\text { (age(M,SD), } \\
\text { sex) }\end{array}$ & Study aims & $\begin{array}{l}\text { Intervention } \\
\text { (cognitive- } \\
\text { motor task) }\end{array}$ & $\begin{array}{l}\text { Cognitive task and } \\
\text { measurement }\end{array}$ & Motor task and measurement & $\begin{array}{l}\text { Training } \\
\text { duration }\end{array}$ \\
\hline \multicolumn{7}{|c|}{ Randomised controlled trials } \\
\hline $\begin{array}{l}\text { Azadian } \\
(2016)[26]\end{array}$ & $\begin{array}{l}N=30 \\
\text { IG1:n=10, } \\
73.9 \pm 5.5 \\
\text { n.a. } \\
\text { IG } 2: n=10 \\
73.8 \pm 3.9 \\
\text { n.a. } \\
\text { CG: } n=10 \\
73.7 \pm 4.4 \\
\text { n.a. }\end{array}$ & $\begin{array}{l}\text { evaluation of the effect of } \\
\text { two cognitive training } \\
\text { methods on pattern of gait }\end{array}$ & $\begin{array}{l}\text { IG1: GDT } \\
\text { IG2: CT } \\
\text { CG: - }\end{array}$ & $\begin{array}{l}\text { INH/PS (A-mo/Vi-mo): } \\
\text { Reaction time to auditory } \\
\text { stimulus/visual stimulus on } \\
\text { screen; } \\
\text { WM: Wechsler Adult } \\
\text { Intelligence Scale, Digit } \\
\text { Symbol B/F }\end{array}$ & $\begin{array}{l}\text { walking ST and DT condition: } \\
\text { Vicon System, asymmetry } \\
\text { index (AS) }\end{array}$ & $\begin{array}{l}18 \mathrm{~h} / \\
3 \times 45 \\
\text { min p.w. } \\
\text { for } 8 \text { wk. }\end{array}$ \\
\hline $\begin{array}{l}\text { Bacha (2018) } \\
\text { [32] }\end{array}$ & $\begin{array}{l}N=46 \\
\text { IG: } n=23 \\
8 \mathrm{~m} / 15 f \\
\text { CG: } n=23 \\
4 \mathrm{~m} / 19 f\end{array}$ & $\begin{array}{l}\text { effectiveness of KAG games } \\
\text { versus CPT } \\
\text { to improve postural control, } \\
\text { gait, fitness and cognition }\end{array}$ & $\begin{array}{l}\text { IG1: EXG } \\
\text { CG: PHY }\end{array}$ & GF: MoCa & $\begin{array}{l}\text { Postural Control: Mini-BESTest; } \\
\text { Gait: FGA } \\
\text { Fitness: } 6 \text { MST }\end{array}$ & $\begin{array}{l}14 \mathrm{~h} \\
2 \times 60 \\
\text { min p.w } \\
\text { for } 7 \text { wk. }\end{array}$ \\
\hline $\begin{array}{l}\text { Eggenberger } \\
\text { (2015) [27] }\end{array}$ & $\begin{array}{l}N=89 \\
\text { IG1:n=30, } \\
77.3 \pm 6.3 \\
\text { n.a. } \\
\text { IG }: n=29 \\
78.5 \pm 5.1 \\
\text { n.a. IG3:n= } \\
\text { 30, } 80.8 \pm \\
\text { 4.7,n.a. }\end{array}$ & $\begin{array}{l}\text { comparison of effects of } \\
\text { physical MCT training to a } \\
\text { stepping-based Exergame on } \\
\text { cognition }\end{array}$ & $\begin{array}{l}\text { IG1:GDT-EXG } \\
\text { (Stepmania) } \\
\text { IG2:GDT- EXG } \\
\text { (Memory) } \\
\text { IG3:PHY } \\
\text { (Walking) }\end{array}$ & $\begin{array}{l}\text { EF/SH(vi-mo):TMT-B } \\
\text { WM: Paired-Associates } \\
\text { Learning Task; ATT: Age } \\
\text { concentration test A and B; } \\
\text { PS (vi-mo) TMT-A, DSST } \\
\text { and WAIS-R; GCF: MoCA }\end{array}$ & n.a. & $\begin{array}{l}36 \mathrm{~h} / \\
3 \times 60 \\
\text { min p.w. } \\
\text { for } 312 \\
\text { wk. / } 24 \\
\text { wk. }\end{array}$ \\
\hline $\begin{array}{l}\text { Eggenberger } \\
\text { (2016) [33] }\end{array}$ & $\begin{array}{l}N=33 \\
\text { IG: } n=19 \\
72.8 \pm 5.9,7 \\
\mathrm{~m} / 12 \mathrm{f} \\
\text { CG: } n=14 \\
77.8 \pm 7.4,5 \\
\mathrm{~m} / 9 f\end{array}$ & $\begin{array}{l}\text { effects of DT video game } \\
\text { against conventional balance } \\
\text { training on PFC activity } \\
\text { during walking and on EF }\end{array}$ & $\begin{array}{l}\text { IG: GDT- EXG } \\
\text { (Stepmania) } \\
\text { CG:ST } \\
\text { (Balance) }\end{array}$ & $\begin{array}{l}\text { EF/SH (vi-ma) TMT-B } \\
\text { ATT/ INH (vi-ma) Stroop } \\
\text { test } \\
\text { GCF: MoCA }\end{array}$ & $\begin{array}{l}\text { lower extremity function: } \\
\text { SPPB }\end{array}$ & $\begin{array}{l}18 \mathrm{~h} / \\
3 \times 30 \\
\min p . w \\
\text { for } 12 \\
\text { wk. }\end{array}$ \\
\hline $\begin{array}{l}\text { Falbo (2016) } \\
{[15]}\end{array}$ & $\begin{array}{l}N=36 \\
\text { IG: } n=20 \\
71.5 \pm 6.7,2 \\
\mathrm{~m} / 18 \mathrm{f} \\
\text { CG: } n=16 \\
73.7 \pm 4.5,2 \\
\mathrm{~m} / 14 \mathrm{f}\end{array}$ & $\begin{array}{l}\text { benefits of a DT training } \\
\text { specifically on EF compared } \\
\text { to physical training with } \\
\text { lower executive demands }\end{array}$ & $\begin{array}{l}\text { IG: GDT } \\
C T: S T\end{array}$ & $\begin{array}{l}\text { EF (A-ve): verbal RNG Test } \\
\text { INH (A-ve) Turning point } \\
\text { index, adjacency, runs } \\
\text { WM (A-ve): redundancy, } \\
\text { coupon, repetition gap }\end{array}$ & $\begin{array}{l}\text { walking ST and DT: speed, } \\
\text { gait length and cadence } \\
\text { recording with photocell } \\
\text { system }\end{array}$ & $\begin{array}{l}24 \mathrm{~h} / \\
2 \times 60 \\
\min p . w . \\
\text { for } 12 \\
\text { wk. }\end{array}$ \\
\hline $\begin{array}{l}\text { Hars (2014) } \\
{[34]}\end{array}$ & $\begin{array}{l}N=134 \\
\text { IG: } n=66 \\
75 \pm 8,2 \mathrm{~m} / \\
64 f \\
\text { CG: } n=68 \\
76 \pm 6,3 \mathrm{~m} / \\
65 f\end{array}$ & $\begin{array}{l}\text { effects of a multi-task music } \\
\text { based training on cognitive } \\
\text { functioning and mood }\end{array}$ & $\begin{array}{l}\text { IG: GDT } \\
\text { CG: - }\end{array}$ & $\begin{array}{l}\text { GCF (vi-ma): MMSE, CDT, } \\
\text { FAB }\end{array}$ & n.a. & $\begin{array}{l}25 \mathrm{~h} / \\
2 \times 30 \\
\text { min p.w. } \\
\text { for } 25 \\
\text { wk. }\end{array}$ \\
\hline $\begin{array}{l}\text { Hiyamizu } \\
\text { (2012) [35] }\end{array}$ & $\begin{array}{l}N=43 \\
\text { IG: } n=21 \\
71.2 \pm 4.4,5 \\
\mathrm{~m} / 16 \mathrm{f} \\
\text { CG: } n=22 \\
72.9 \pm 5.1 \\
12 \mathrm{~m} / 10 \mathrm{f}\end{array}$ & $\begin{array}{l}\text { effects of a DT balance } \\
\text { training on postural control } \\
\text { while performing a cognitive } \\
\text { task }\end{array}$ & $\begin{array}{l}\text { IG: GDT } \\
\text { CG:ST } \\
\text { (balance and } \\
\text { strength) }\end{array}$ & $\begin{array}{l}\text { EF (vi-ma): TMT (B-A) } \\
\text { INH/ATT (vi-ve): Stroop task }\end{array}$ & $\begin{array}{l}\text { Standing and walking, Chair } \\
\text { Standing Test, TUGT, } \\
\text { Functional Reach Test, COP } \\
\text { displacements }\end{array}$ & $\begin{array}{l}24 \mathrm{~h} / \\
2 \times 60 \\
\text { p.w. for } \\
12 \text { wk. }\end{array}$ \\
\hline $\begin{array}{l}\text { Kitazawa } \\
\text { (2015) [36] }\end{array}$ & $\begin{array}{l}N=60 \\
\mathrm{G}: n=30 \\
76.8 \pm 4.4 \\
17 \mathrm{~m} / 13 \mathrm{f} \\
\mathrm{CG}: n=30 \\
75.5 \pm 3.7 \\
10 \mathrm{~m} / 20 \mathrm{f}\end{array}$ & $\begin{array}{l}\text { effect of a net-stepping exer- } \\
\text { cise on cognitive perform- } \\
\text { ance and gait function }\end{array}$ & $\begin{array}{l}\text { IG: GDT } \\
\text { CG: - }\end{array}$ & $\begin{array}{l}\text { GCF: Touch Panel-Type } \\
\text { Demetia Assesment Scale } \\
\text { (TDAS) }\end{array}$ & Mobility: TUG & $\begin{array}{l}8 \mathrm{~h} / \\
1 \times 60 \\
\min \text { p.w. } \\
\text { for } 8 \text { wk. }\end{array}$ \\
\hline
\end{tabular}


Table 1 Characteristics of included studies (Continued)

\begin{tabular}{|c|c|c|c|c|c|c|}
\hline $\begin{array}{l}\text { Eligible author } \\
\text { and year }\end{array}$ & $\begin{array}{l}\text { Participants } \\
\text { (age(M,SD), } \\
\text { sex) }\end{array}$ & Study aims & $\begin{array}{l}\text { Intervention } \\
\text { (cognitive- } \\
\text { motor task) }\end{array}$ & $\begin{array}{l}\text { Cognitive task and } \\
\text { measurement }\end{array}$ & Motor task and measurement & $\begin{array}{l}\text { Training } \\
\text { duration }\end{array}$ \\
\hline $\begin{array}{l}\text { MacLean } \\
\text { (2014) [37] }\end{array}$ & $\begin{array}{l}N=45 \\
I G 1: n=15 \\
73.2 \pm 5.36 \\
4 \mathrm{~m} / 11 \mathrm{f} \\
\mathrm{IG} 2: n=15 \\
69.1 \pm 3.37 \\
6 \mathrm{~m} / 9 \mathrm{f} \\
\mathrm{IG} 3: n=15 \\
72.9 \pm 6.49 \\
7 \mathrm{~m} / 8 \mathrm{f}\end{array}$ & $\begin{array}{l}\text { effects of musical training on } \\
\text { the gait and cognition in } \\
\text { healthy older people }\end{array}$ & $\begin{array}{l}\text { IG1: GDT } \\
\text { IG2: ST } \\
\text { IG3: ST }\end{array}$ & $\begin{array}{l}\text { GCF: MMSE; } \\
\text { EF/ SH/ PS: TMT A/B; } \\
\text { WM (ve) DS b/f; Immediate } \\
\text { and delayed story recall }\end{array}$ & $\begin{array}{l}\text { Gait (ST/DT conditions): } \\
\text { velocity, stability }\end{array}$ & n.a. \\
\hline $\begin{array}{l}\text { Malliot (2012) } \\
{[29]}\end{array}$ & $\begin{array}{l}N=32 \\
\text { IG } n=16 \\
73.47 \pm 4.10 \\
C G n=16 \\
73.47 \pm 3.0 \\
5 \mathrm{~m} / 27 f\end{array}$ & $\begin{array}{l}\text { determine whether } \\
\text { exergame training sport } \\
\text { activity would show transfer } \\
\text { to cognitive functions }\end{array}$ & $\begin{array}{l}\text { IG:GDT- EXG } \\
\text { CG: - }\end{array}$ & $\begin{array}{l}\text { EF/INH/ ATT (vi-ve): Stroop } \\
\text { Test, TMT A/B. Matrix } \\
\text { Reasoning Test; } \\
\text { WM (vi-ve): Spatial Span } \\
\text { Test b/f, Directional } \\
\text { Headings, Mental Rotation } \\
\text { Letter Digit; } \\
\text { PS: cancellation test, } \\
\text { number comparison test; } \\
\text { PS(vi-ma): Reaction time } \\
\text { test, Plate Tapping test }\end{array}$ & $\begin{array}{l}\text { Heart rate ( } 6 \text { min walking); } \\
\text { Chair Stand } \\
\text { 8-ft-up and go; Arm curls, } \\
\text { "back scratch" }\end{array}$ & $\begin{array}{l}12 \mathrm{~h} / \\
1 \times 60 \\
\text { min p.w. } \\
\text { for } 12 \\
\text { wk. }\end{array}$ \\
\hline $\begin{array}{l}\text { Nishiguchi } \\
\text { (2015) [38] }\end{array}$ & $\begin{array}{l}N=48 \\
\text { IG: } n=24 \\
73.0 \pm 4.8 \\
13 \mathrm{~m} / 11 \mathrm{fm} \\
\text { CG: } n=24 \\
73.5 \pm 5.6 \\
13 \mathrm{~m} / 11 \mathrm{f}\end{array}$ & $\begin{array}{l}\text { investigate whether a } \\
\text { physical and cognitive } \\
\text { program can improve } \\
\text { cognitive function and brain } \\
\text { activation efficiency in older } \\
\text { people }\end{array}$ & $\begin{array}{l}\text { IG: GDT } \\
\text { CG: - }\end{array}$ & $\begin{array}{l}\text { GCF: MMSE } \\
\text { WM: WMS-R } \\
\text { EF/ATT PS: (vi): TMT B-A, } \\
\text { Go-No-Go-Stimulus, Test off } \\
\text { attentional control }\end{array}$ & $\begin{array}{l}\text { 10-m walking test, TUGT, 5- } \\
\text { CtST }\end{array}$ & $\begin{array}{l}18 \mathrm{~h} / \\
1 \times 90 \\
\text { minp.w. } \\
\text { for } 12 \\
\text { wk. }\end{array}$ \\
\hline $\begin{array}{l}\text { Ordnung } \\
\text { (2017) [30] }\end{array}$ & $\begin{array}{l}N=30 \\
\text { IG: } n=15 \\
69 \pm 79 \\
6.34, \text { n.a. } \\
\text { CG: } n=15 \\
68 \pm 4.67 \\
\text { n.a. }\end{array}$ & $\begin{array}{l}\text { investigate the effect of a } \\
\text { whole-body Exergame train- } \\
\text { ing intervention }\end{array}$ & $\begin{array}{l}\text { IG: GDT- EXG } \\
\text { CG:- }\end{array}$ & $\begin{array}{l}\text { ATT/PS: Test of Attentional } \\
\text { Performance; } \\
\text { WM (vi-ma): n-back task 2; } \\
\text { INH: Go/ No-Go-Stimulus }\end{array}$ & $\begin{array}{l}3 \text { min. Step Test, upper body } \\
\text { muscle endurance test, grip } \\
\text { strength, hand motor skills; } \\
\text { Motor RT: Ruler Drop Test, } \\
\text { Balance (Balance Board) }\end{array}$ & $\begin{array}{l}12 \mathrm{~h} / \\
2 \times 60 \\
\text { min p.w. } \\
\text { for } 6 \mathrm{wk} .\end{array}$ \\
\hline $\begin{array}{l}\text { Schaettin } \\
\text { (2016) [39] }\end{array}$ & $\begin{array}{l}N=27 \\
\text { Mean } 80 \\
\text { IG1: } n=13 \\
8 \mathrm{~m} / 5 f \\
\text { IG } 2: n=14 \\
80,7 \mathrm{~m} / 7 \mathrm{f}\end{array}$ & $\begin{array}{l}\text { compare Exergame training } \\
\text { with conventional balance } \\
\text { training }\end{array}$ & $\begin{array}{l}\text { IG1: SDT- } \\
\text { EXG } \\
\text { IG2: Balance }\end{array}$ & $\begin{array}{l}\text { EF (vi-ma):computerized } \\
\text { TAP (WM, SHI, divided ATT, } \\
\text { INH (Go-/No-Go-task)) }\end{array}$ & n.a. & $\begin{array}{l}15 \mathrm{~h} / \\
3 \times 30 \\
\min \mathrm{p} . \mathrm{w} \text {. } \\
\text { for } 10 \\
\text { wk. }\end{array}$ \\
\hline $\begin{array}{l}\text { Schoene } \\
\text { (2013) [31] }\end{array}$ & $\begin{array}{l}N=37 \\
\text { IG: } n=15 \\
77.5 \pm 4.5 \\
\text { n.a. } \\
\text { CG; } n=17, \\
78.4 \pm 4.5 \\
\text { n.a. }\end{array}$ & $\begin{array}{l}\text { effects of a stepping exergae } \\
\text { on stepping performance } \\
\text { and associated fall risk }\end{array}$ & $\begin{array}{l}\text { IG: SDT- EXG } \\
\text { CG: - }\end{array}$ & $\begin{array}{l}\text { PS (vi-mo): Choice } \\
\text { Stepping Reaction time; } \\
\text { EF: TMT; } \\
\text { DTC:TUG-DT }\end{array}$ & $\begin{array}{l}\text { Physiological Profile } \\
\text { Assessment (Fall Risk); TUGT, } \\
\text { 5STS, Alternate Step Test (AST }\end{array}$ & $\begin{array}{l}16 \mathrm{~h} / \\
3 \times 20 \\
\text { min p.w. } \\
\text { for } 16 \\
\text { wk. }\end{array}$ \\
\hline $\begin{array}{l}\text { Schoene } \\
\text { (2015) [40] }\end{array}$ & $\begin{array}{l}N=90 \\
\text { IG: } n=47 \\
82 \pm 7,66 \% f \\
\text { CG: } n=43 \\
81 \pm 7,67 \% f\end{array}$ & $\begin{array}{l}\text { effectiveness of step-based } \\
\text { exercise game on cognitive } \\
\text { functions associated with falls }\end{array}$ & $\begin{array}{l}\text { IG: SDT- EXG } \\
\text { CG: } \\
\text { education } \\
\text { brochure }\end{array}$ & $\begin{array}{l}\text { EF(vi-mo): Stroop Stepping } \\
\text { Test (SST); } \\
\text { PS/ATT (vi-mo): Letter-digit } \\
\text { test, CRT + CSRT; TMT A, At- } \\
\text { tentional network test; } \\
\text { INH: Victoria Stroop Task } \\
\text { DTC:TUG-DT; WM: Digit } \\
\text { span B; }\end{array}$ & n.a. & $\begin{array}{l}16 \mathrm{~h} / / \\
3 \times 20 \\
\min \mathrm{p} . \mathrm{w} \text {. } \\
\text { for } 16 \\
\text { wk. }\end{array}$ \\
\hline
\end{tabular}


Table 1 Characteristics of included studies (Continued)

\begin{tabular}{|c|c|c|c|c|c|c|}
\hline $\begin{array}{l}\text { Eligible author } \\
\text { and year }\end{array}$ & $\begin{array}{l}\text { Participants } \\
\text { (age(M,SD), } \\
\text { sex) }\end{array}$ & Study aims & $\begin{array}{l}\text { Intervention } \\
\text { (cognitive- } \\
\text { motor task) }\end{array}$ & $\begin{array}{l}\text { Cognitive task and } \\
\text { measurement }\end{array}$ & Motor task and measurement & $\begin{array}{l}\text { Training } \\
\text { duration }\end{array}$ \\
\hline $\begin{array}{l}\text { Wollesen } \\
\text { (2017a) [41] }\end{array}$ & $\begin{array}{l}N=95 \\
\text { IG1: } n=26 \\
72.2 \pm 4.6 \\
10 \mathrm{~m} / 16 \mathrm{f} \\
\text { IG }: n=30 \\
69.8 \pm 5.7,2 \\
\mathrm{~m} / 28 \mathrm{f} \\
\mathrm{CG} 1: n=19, \\
72.9 \pm 4.4 \\
\mathrm{~m} / 12 \mathrm{f} \\
\mathrm{CG} 2: n=20 \\
72.7 \pm 5.3,3 \\
\mathrm{~m} / 17 f\end{array}$ & $\begin{array}{l}\text { effects of a DT training in } \\
\text { people with and without } \\
\text { concern about falling on } \\
\text { walking performance }\end{array}$ & $\begin{array}{l}\text { IG1: GDT } \\
(\text { FES-I }<20) \\
\text { IG2: GDT } \\
(\text { FES-I }>20) \\
\text { CG1: - (FES- } \\
\text { I<20) } \\
\text { CG2:- (FES- } \\
\text { I> 20) }\end{array}$ & $\begin{array}{l}\text { DT/ATT performance (vi- } \\
\text { ve): Stroop Task }\end{array}$ & $\begin{array}{l}\text { Walking performance: } \\
\text { ST =30-s walking test } \\
\text { DT: } 30 \text {-s-walking test + (vi-ve) } \\
\text { Stroop Task }\end{array}$ & $\begin{array}{l}12 \mathrm{~h} 1 \times \\
60 \mathrm{~min} \\
\text { p.w. for } \\
12 \mathrm{wk} \text {. }\end{array}$ \\
\hline $\begin{array}{l}\text { Wollesen } \\
\text { (2017b) [42] }\end{array}$ & $\begin{array}{l}N=78 \\
\text { IG1: } n=29 \\
70.7 \pm 4.9,7 \\
m / 22 f \\
\text { IG2: } n=23 \\
71.7 \pm 4.9,8 \\
m / 15 f \\
\text { CG: } n=26 \\
73.7 \pm 5.0,7 \\
m / 19 f\end{array}$ & $\begin{array}{l}\text { effects of a DT } \\
\text { balancetraining and a ST } \\
\text { strength and resistance } \\
\text { training on motor } \\
\text { performance during DT } \\
\text { walking }\end{array}$ & $\begin{array}{l}\text { IG1: GDT } \\
\text { IG2: PHY } \\
\text { (Strength } \\
\text { and } \\
\text { resistance) } \\
\text { CG: - }\end{array}$ & $\begin{array}{l}\text { ATT/DT performance (au- } \\
\text { ve): Stroop task while } \\
\text { walking }\end{array}$ & $\begin{array}{l}\text { Walking performance: ST and } \\
\text { DT conditions }\end{array}$ & $\begin{array}{l}12 \mathrm{~h} / \\
1 \times 60 \\
\min \text { p.w. } \\
\text { for } 12 \\
\text { wk. }\end{array}$ \\
\hline $\begin{array}{l}\text { You (2009) } \\
{[43]}\end{array}$ & $\begin{array}{l}N=13 \\
\text { IG: } n=8 \\
70.5 \pm 6.8,2 \\
\mathrm{~m} / 11 \mathrm{f} \\
\text { CG: } n=5 \\
68.0 \pm 3.3,1 \\
\mathrm{~m} / 4 \mathrm{f}\end{array}$ & $\begin{array}{l}\text { determine long-term practice } \\
\text { effects of CGI on cognition } \\
\text { and gait performance in } \\
\text { older people with a history } \\
\text { of falls }\end{array}$ & $\begin{array}{l}\text { IG: SDT } \\
\text { CG: ST } \\
\text { (walking) }\end{array}$ & $\begin{array}{l}\text { Memory recall (ve): } \\
\text { memorise and recall words }\end{array}$ & $\begin{array}{l}\text { walking performance under } \\
\text { DT conditions: velocity; } \\
\text { stability; COP displacements }\end{array}$ & $\begin{array}{l}15 \mathrm{~h} / \\
5 \times 30 \\
\text { min p.w. } \\
\text { for } 6 \text { wk. }\end{array}$ \\
\hline \multicolumn{7}{|c|}{ Controlled clinical trials and other } \\
\hline $\begin{array}{l}\text { Ansai (2017) } \\
{[25]}\end{array}$ & $\begin{array}{l}N=80 \\
\text { IG: } n=41 \\
68.5 \pm 8.4 \\
\text { n.a. } \\
\text { CG: } n=39 \\
68.5 \pm 6.3 \\
\text { n.a. }\end{array}$ & $\begin{array}{l}\text { effects of the addition of a } \\
\text { dual task to MCT on } \\
\text { cognition }\end{array}$ & $\begin{array}{l}\text { IG: TDT } \\
\text { (MCT + CT), } \\
\text { CG: MCT }\end{array}$ & $\begin{array}{l}\text { GCF: MMSE, MoCA; } \\
\text { VS: CDT; EF (Ve-mo): } \\
\text { DTC: TUGT-DT memorising } \\
\text { number and dialling while } \\
\text { walking }\end{array}$ & TUGT: mobility & $\begin{array}{l}60 \mathrm{~h} / \\
3 \times 50 \\
\text { min p.w. } \\
\text { for } 12 \\
\text { wk. }\end{array}$ \\
\hline $\begin{array}{l}\text { Bisson (2007) } \\
{[44]}\end{array}$ & $\begin{array}{l}N=24 \\
\text { IG1: } n=12 \\
74 . \pm 3.6,7 \\
\mathrm{~m} / 5 f \\
\text { IG }: n=12 \\
74 \pm 4.92,2 \\
\mathrm{~m} / 9 f\end{array}$ & $\begin{array}{l}\text { determine the effect of VR } \\
\text { and } \mathrm{BF} \text { training on balance } \\
\text { and reaction time in older } \\
\text { people }\end{array}$ & $\begin{array}{l}\text { IG1: GDT- } \\
\text { EXG (VR) } \\
\text { IG2: GDT- } \\
\text { EXG (BF) }\end{array}$ & $\begin{array}{l}\text { PS (au-ve): verbal reaction } \\
\text { to auditory cue }\end{array}$ & $\begin{array}{l}\text { COP displacement; CB\&M } \\
\text { Scale }\end{array}$ & $\begin{array}{l}10 \mathrm{~h} / \\
2 \times 30 \\
\text { min p.w. } \\
\text { for } 10 \\
\text { wk. }\end{array}$ \\
\hline $\begin{array}{l}\text { Chuang } \\
\text { (2015) [45] }\end{array}$ & $\begin{array}{l}N=26 \\
\text { IG1: } n=7 \\
69.43 \pm 3.82 \\
\text { IG } 2: n=11 \\
67.01 \pm 1.67 \\
\text { CG: } n=8 \\
68.25 \pm 3.96 \\
26 f / 0 m\end{array}$ & $\begin{array}{l}\text { examine whether DDR } \\
\text { training would exert similar } \\
\text { effect on interference control } \\
\text { as that brisk walking in } \\
\text { elderly individual }\end{array}$ & $\begin{array}{l}\text { IG1: SDT- } \\
\text { EXG (DDR) } \\
\text { IG2: ST } \\
\text { (walking) } \\
\text { CG: - }\end{array}$ & $\begin{array}{l}\text { EF }(V i-m o) \text { : reaction to } \\
\text { visual stimulus on screen in } \\
\text { reaction time/ms; EEG } \\
\text { recording } \\
\text { INH: Flanker Test }\end{array}$ & n.a. & $\begin{array}{l}18 \mathrm{~h} / \\
3 \times 30 \\
\text { min p.w. } \\
\text { for } 12 \\
\text { wk. }\end{array}$ \\
\hline $\begin{array}{l}\text { Heiden } \\
\text { (2010) [46] }\end{array}$ & $\begin{array}{l}N=16 \\
\text { Mean Age } \\
77 \\
\text { IG } n=9,5 \\
\mathrm{f} / 4 \mathrm{~m} \\
C G=7,6 \mathrm{f} / \\
1 \mathrm{~m}\end{array}$ & $\begin{array}{l}\text { Effects of a games-based bal- } \\
\text { ance training program on } \\
\text { general fitness and atten- } \\
\text { tional demands in postural } \\
\text { control }\end{array}$ & $\begin{array}{l}\text { IG: GDT } \\
\text { CT: - }\end{array}$ & $\begin{array}{l}\text { PS }(\mathrm{A}-\mathrm{Ve}) \text { : reaction on } \\
\text { auditory simulus }\end{array}$ & $\begin{array}{l}\text { CB\&M Scale, } 6 \text { min walking, } \\
\text { COP displacements (RMS) }\end{array}$ & $\begin{array}{l}8 \mathrm{~h} / 2 \times \\
30 \mathrm{~min} \\
\text { p.w. for } \\
8 \mathrm{wk} \text {. }\end{array}$ \\
\hline
\end{tabular}


Table 1 Characteristics of included studies (Continued)

\begin{tabular}{|c|c|c|c|c|c|c|}
\hline $\begin{array}{l}\text { Eligible author } \\
\text { and year }\end{array}$ & $\begin{array}{l}\text { Participants } \\
\text { (age(M,SD), } \\
\text { sex) }\end{array}$ & Study aims & $\begin{array}{l}\text { Intervention } \\
\text { (cognitive- } \\
\text { motor task) } \\
\end{array}$ & $\begin{array}{l}\text { Cognitive task and } \\
\text { measurement }\end{array}$ & Motor task and measurement & $\begin{array}{l}\text { Training } \\
\text { duration }\end{array}$ \\
\hline $\begin{array}{l}\text { Kayama } \\
(2014)[28]\end{array}$ & $\begin{array}{l}n=41 ;>65 \\
\text { IG: } n=26, \\
\text { n.a. } \\
\text { CG: } n=15, \\
\text { n.a. }\end{array}$ & $\begin{array}{l}\text { whether or not a DT Thai Chi } \\
\text { training program would } \\
\text { effectively improve cognitive } \\
\text { functions }\end{array}$ & $\begin{array}{l}\text { IG: ST+ EXG } \\
\text { (GDT Thai } \\
\text { Chi) } \\
\text { CG: } \\
\text { Standardised } \\
\text { Training }\end{array}$ & $\begin{array}{l}\text { EF/PS: TMT A/B } \\
\text { VF (ve): Verbal Fluency Task }\end{array}$ & n.a. & $\begin{array}{l}60 \mathrm{~h} / \\
1 \times 80 \\
\text { min p.w. } \\
\text { for } 12 \\
\text { wk. }\end{array}$ \\
\hline $\begin{array}{l}\text { Morita (2018) } \\
{[47]}\end{array}$ & $\begin{array}{l}N=19 \\
\text { IG: } n=8, \\
75.0 \pm 1.5,8 \\
\text { m, } \\
\text { CG: } n=11 \\
71.9 \pm 4.0,2 \\
m / 9 f\end{array}$ & $\begin{array}{l}\text { effect of 2-year cognitive- } \\
\text { motor dual-task (DT) training } \\
\text { on cognitive functions and } \\
\text { motor ability }\end{array}$ & $\begin{array}{l}\text { IG: GDT } \\
\text { CG: - }\end{array}$ & $\begin{array}{l}\text { GCF: Modified Mini-Mental } \\
\text { State(3MS) } \\
\text { PS/ATT (vi): TMT A/B }\end{array}$ & $\begin{array}{l}\text { Quadriceps isometric muscle } \\
\text { strength motor ability: TUGT, } \\
\text { maximal step length (MSL) }\end{array}$ & $\begin{array}{l}104 \mathrm{~h} / \\
1 \times 60 \\
\text { min p.w. } \\
\text { for } 104 \\
\text { wk }\end{array}$ \\
\hline $\begin{array}{l}\text { Theill (2013) } \\
\text { [48] }\end{array}$ & $\begin{array}{l}N=63 \\
\text { IG1: } n=21 \\
72.39 \pm 4.19 \\
\text { IG2: } n=16 \\
73.33 \pm 6.08 \\
\text { CG: } n=26 \\
70.90 \pm 4.77\end{array}$ & $\begin{array}{l}\text { effects of simultaneously } \\
\text { performed WM and PHY } \\
\text { training on cognitive and } \\
\text { motor-cognitive dual task } \\
\text { performance }\end{array}$ & $\begin{array}{l}\text { IG1: GDT } \\
\text { IG2: ST } \\
\text { (cognitive } \\
\text { training) } \\
\text { CG: - }\end{array}$ & $\begin{array}{l}\text { paired associates learning; } \\
\text { ATT (vi-ma): continuous } \\
\text { performance task } \\
\text { EF:sequential learning } \\
\text { PS(vi-ma): Digit-letter task } \\
\text { WM (ve): n-nack task }\end{array}$ & Walking ST/DT conditions & $\begin{array}{l}13 \mathrm{~h} / \\
2 \times 40 \\
\text { min p.w. } \\
\text { for } 10 \\
\text { wk. }\end{array}$ \\
\hline
\end{tabular}

Legend: Participants: IG intervention group, CG control group, $f$ female, $m$ male, MMSE Mini Mental Status Examination, MoCA Montreal Cognitive Assesment; Intervention: $C L$ Cognitive load, KAG kinect adventures games, $C P T$ conventional physical therapy, MT Motor task, MCT Multicomponent Training, DT Dual Tasking, ST Single Tasking, GDT General Dual-Tasking, SDT Specific Dual-Tasking, EXG Exergaming, CT Computerized training, VR Virtual Reality, BF Biofeedback, PHY Physical Training; Cognitive assessment: GCF Global cognitive function, PS Processing Speed, Sh Shifting, EF Executive Function, INH Inhibition, WM Working Memory, ATT Attention, VS Visuospatial, DTC Dual-Task Costs, Stimulus-response: A-Ve Auditory Verbal, $A$-ma auditory manual, Vi-Ve Visual-verbal, Vi-mo Visualmotor, Ve-mo Verbal-motor, Assessments: TUGT-DT Timed up and go test with dual tasking, motor task, TUGT Timed up and go test, TMT A/B Trail Marking test A/ B, DS B/F Digit Symbol backwards/forwards, CSRT Choice Stepping Reaction Time, Training duration: p.w. per week., wk week

virtual environment, which could be moved or controlled by bodily movements. A cognitive load was also provided, when the intervention required the participant to work with a visual feedback or biofeedback as part of the technology-based game.

The cognitive-motor components differed between the evaluated studies. Fourteen trials used a traditional dualtask intervention and eleven trials used modern technology or a combination of traditional and technology-based interventions. The included studies show a range of interventions, which differed in both motor exercise and cognitive load of the additional cognitive task. Motor interventions varied from specific walking or stepping tasks to general balance and strength training (cf. Table 3). Most trials included different additional cognitive tasks to the motor components (e.g. working memory tasks, verbal fluency tasks, visual search tasks) and can be named as GDT intervention. Five out of 25 studies used a SDT training (e.g. body shifting to control a virtual paddle) to examine the influence of a cognitive-motor training on cognition $[31,39,40,46]$.

\section{Results on cognition}

The intervention results, including effects on global cognition, global executive function, mental flexibility, inhibitory control, working memory, visuospatial planning, verbal fluency, attentional control, processing speed as well as dual-task costs are outlined in Tables 3 and 4.

\section{Effects on global cognitive function}

Seven studies examined the effects of training intervention on global cognitive function $[25,32-34$, $36,38,47]$. All training interventions were conducted as a GDT training and two used the exergaming approach [32, 33].

Five out of seven studies showed positive improvements of global cognitive function tests such as MMSE, MoCA or comparable instruments [32, 34, 36, 38, 47] (cf. Table 3). All but one of these studies used a GDT training method. Although all studies used a walking or stepping motor component, cognitive tasks were heterogeneous. While Hars and colleagues [34] required their participants to adapt their pace to the rhythm of the music, Kitazawa and colleagues [36] used a targeted stepping exercise while participants needed to recite a children's song. Similarly, Nishiguchi and colleagues [38] delivered a walking exercise while conducting a verbal fluency task. Bacha and colleagues [32] showed positive effects on global cognitive function due to an exergame intervention, using the Xbox Kinect system, which required whole body movements for conduction of the virtual game on screen. The two studies that did not show a positive impact on global cognitive function used a GDT. The study, by Ansai and 
Table 2 Quality assessment of included studies according to PEDro scale

\begin{tabular}{|c|c|c|c|c|c|c|c|c|c|c|c|c|}
\hline \multirow[t]{2}{*}{ Study } & \multicolumn{11}{|c|}{ Quality criteria } & \multirow{2}{*}{$\begin{array}{l}\text { Quality } \\
\text { Score }\end{array}$} \\
\hline & 1 & 2 & 3 & 4 & 5 & 6 & 7 & 8 & 9 & 10 & 11 & \\
\hline Ansai [25] & $x$ & - & - & - & - & - & - & - & $x$ & $x$ & $x$ & 4 \\
\hline Azadian [26] & - & $x$ & - & $x$ & - & - & - & $x$ & $x$ & $x$ & $x$ & 6 \\
\hline Bacha [32] & $x$ & $x$ & $x$ & $x$ & - & - & - & $x$ & $x$ & - & $x$ & 7 \\
\hline Bisson [44] & $x$ & - & - & - & - & $x$ & - & - & - & $x$ & $x$ & 4 \\
\hline Chuang [45] & $x$ & - & - & $x$ & - & - & - & $x$ & $x$ & $x$ & $x$ & 6 \\
\hline Eggenberger 2015 [27] & $x$ & $x$ & - & $x$ & $x$ & - & - & $x$ & $x$ & $(\mathrm{x})$ & $x$ & 7 \\
\hline Eggenberger 2016 [33] & $x$ & $x$ & $x$ & $x$ & $x$ & - & - & - & $x$ & $x$ & $x$ & 8 \\
\hline Falbo [15] & $x$ & $x$ & - & - & - & - & - & - & $x$ & - & $x$ & 4 \\
\hline Hars [34] & $x$ & $x$ & - & $x$ & - & - & $x$ & - & $x$ & $x$ & $x$ & 7 \\
\hline Heiden [46] & $x$ & - & - & - & - & - & - & $(x)$ & - & $x$ & $x$ & 3 \\
\hline Hiyamizu [35] & $x$ & $x$ & $(x)$ & $(x)$ & - & $x$ & - & - & - & $x$ & $x$ & 5 \\
\hline Kayama [28] & $x$ & - & - & - & - & - & - & $x$ & - & $(\mathrm{x})$ & $x$ & 3 \\
\hline Kitazawa [36] & $x$ & $(x)$ & - & $x$ & - & - & - & $x$ & $x$ & $x$ & $x$ & 6 \\
\hline MacLean [37] & $x$ & $x$ & - & $x$ & - & - & - & - & $x$ & $x$ & $x$ & 6 \\
\hline Maillot [29] & $x$ & $x$ & - & $x$ & - & - & - & $x$ & $x$ & - & $x$ & 6 \\
\hline Morita [47] & $x$ & - & - & $x$ & - & - & $x$ & $x$ & $x$ & - & $x$ & 6 \\
\hline Nishiguchi [38] & $x$ & $x$ & $(x)$ & $x$ & - & $x$ & - & $x$ & $x$ & - & $x$ & 7 \\
\hline Ordnung [30] & $x$ & $x$ & - & $x$ & - & - & - & $x$ & $x$ & $x$ & $x$ & 7 \\
\hline Schaettin 2016 [39] & $x$ & $x$ & - & $x$ & $x$ & - & - & $x$ & $x$ & $x$ & $x$ & 8 \\
\hline Schoene 2013 [31] & $x$ & $x$ & - & $x$ & - & - & - & $x$ & $x$ & $x$ & $x$ & 7 \\
\hline Schoene 2015 [40] & $x$ & $x$ & $(x)$ & $x$ & - & $x$ & $x$ & $(\mathrm{x})$ & $x$ & $x$ & $x$ & 8 \\
\hline Theill [48] & - & - & - & $x$ & - & - & - & - & - & $x$ & $x$ & 3 \\
\hline Wollesen 2017a [41] & $x$ & $x$ & - & $x$ & - & - & - & $x$ & $x$ & $x$ & $x$ & 7 \\
\hline Wollesen 2017b [42] & $x$ & $x$ & - & $x$ & - & - & - & $x$ & $x$ & $x$ & $x$ & 7 \\
\hline You [43] & $x$ & $(x)$ & - & $x$ & - & - & - & $x$ & $x$ & $(x)$ & $x$ & 5 \\
\hline
\end{tabular}

Legend: 1 - eligibility criteria were specified. 2 - participants were randomly allocated to groups. 3 - allocation was concealed. "4 - the groups were similar at baseline regarding the most important prognostic indicators". 5 - there was blinding of all participants. 6 - there was blinding of all therapists who administered the therapy. 7 - there was blinding of all assessors who measured at least one key outcome. " 8 - measures of at least one key outcome were obtained from more than $85 \%$ of the participants initially allocated to groups". " 9 - all participants for whom outcome measures were available received the treatment or control condition as allocated or, where this was not the case, data for at least one key outcome was analyzed by "intention to treat" ". "10 - the results of between-group statistical comparisons are reported for at least one key outcome". "11 - the study provides both point measures and measures of variability for at least one key outcome". x - "yes" score.. "-" - "no" score. (x)- undertaken with general remarks

colleagues, with a total duration of $60 \mathrm{~h}$ (three times per week over 12 weeks), used a combination of a general balance, strength and walking training with a cognitive arithmetic task [25]. Eggenberger and colleagues [33] provided the Dance Dance Revolution GDT exergame without significant changes in general cognitive function with a duration of $18 \mathrm{~h}$ (three times per week $30 \mathrm{~min}$ for 12 weeks).

Six studies could be integrated into the metaanalysis (Fig. 2). Three of the studies had an inactive control group and three had an active control group (cf. Table 3).

Overall, the interventions showed a significant mean difference of 0.60 [95\% CI 0.29-0.90] on global cognitive functioning. This effect was observed in comparison to active (mean difference 0.53 ) and inactive control groups (mean difference 1.0) with no significant difference for the control group conditions (cf. Fig. 2). However, these results were heterogeneous $\left(\mathrm{I}^{2}=60 \%\right)$ due to the effects of the studies by Morita and colleagues [47] and Eggenberger and colleagues [33]. A sensitivity analysis excluding both studies revealed the effect for the comparison of the DT training intervention to an inactive control or active control group did not remain significant.

\section{Effects on inhibitory control}

Eleven studies evaluated training effects on inhibitory control, with six of these studies showing improvements [31, 33, 35, 41, 42]. Hiyamizu and colleagues [35] used a 
Table 3 Data extraction of included studies

\begin{tabular}{|c|c|c|c|c|c|c|c|}
\hline Author & $\begin{array}{l}\text { Training } \\
\text { intervention }\end{array}$ & Motor-component & $\begin{array}{l}\text { Cognitive } \\
\text { component }\end{array}$ & $\begin{array}{l}\text { Cognitive } \\
\text { measurement }\end{array}$ & Progression & $\begin{array}{l}\text { Control } \\
\text { group(s) }\end{array}$ & Results \\
\hline Ansai [25] & GDT & $\begin{array}{l}\text { Warm up, muscle } \\
\text { strengthening, } \\
\text { balance, } \\
\text { coordination, } \\
\text { flexibility }\end{array}$ & $\begin{array}{l}\text { Working } \\
\text { memory, } \\
\text { inhibition }\end{array}$ & $\begin{array}{l}\text { MMSE (main scores } \\
\text { and subscales) } \\
\text { MoCA (main scores } \\
\text { and subscales), } \\
\text { TUG-DT }\end{array}$ & $\begin{array}{l}\text { In the complexity } \\
\text { of the cognitive } \\
\text { task }\end{array}$ & $\begin{array}{l}\text { Physical } \\
\text { exercises } \\
\text { without DT }\end{array}$ & $\begin{array}{l}\text { No differences } \\
\text { between the } \\
\text { groups regarding } \\
\text { the cognitive } \\
\text { outcomes; the } \\
\text { MMSE and the } \\
\text { visuo-spatial test of } \\
\text { the MoCA in- } \\
\text { creased; DTC } \\
\text { decreased }\end{array}$ \\
\hline Azaidian [26] & GDT & $\begin{array}{l}\text { Standing and } \\
\text { shifting center of } \\
\text { gravity } \\
\text { Walking exercises to } \\
\text { the front, backwards } \\
\text { and sides }\end{array}$ & $\begin{array}{l}\text { Working } \\
\text { memory tasks } \\
\text { Verbal fluency } \\
\text { tasks } \\
\text { Visual search } \\
\text { tasks }\end{array}$ & $\begin{array}{l}\text { Reaction time while } \\
\text { (1) sitting, (2) } \\
\text { standing, (3) } \\
\text { walking, (4) } \\
\text { selective (respond } \\
\text { to direction of task) } \\
\text { Stop Signal Task to } \\
\text { measure inhibitory } \\
\text { control } \\
\text { Working memory } \\
\text { with Wechsler Adult } \\
\text { Intelligence scale } \\
\text { and Digital Symbol } \\
\text { substitution test }\end{array}$ & $\begin{array}{l}\text { Session 1-6 only } \\
\text { motor training } \\
\text { Session } 7-12 \text { motor } \\
\text { training with } \\
\text { simple cognitive } \\
\text { tasks } \\
\text { Session 13-24 } \\
\text { Task complexity } \\
\text { increased }\end{array}$ & $\begin{array}{l}\text { CG 1: } \\
\text { computer- } \\
\text { based EF } \\
\text { training } \\
\text { CG 2: no } \\
\text { intervention }\end{array}$ & $\begin{array}{l}\text { GDT training only } \\
\text { improved the } \\
\text { Wechsler forward in } \\
\text { comparison to } \\
\text { control groups } \\
\text { EF training } \\
\text { improved SST } \\
\text { correct answers } \\
\text { and wrong } \\
\text { answers; stride } \\
\text { asymmetry while } \\
\text { DT walking }\end{array}$ \\
\hline Bacha [32] & $\begin{array}{l}\text { GDT-EX } \\
\text { Xbox Kinect } \\
\text { adventure } \\
\text { game }\end{array}$ & $\begin{array}{l}\text { Fast multidirectional } \\
\text { movements (steps, } \\
\text { squats, jumps, } \\
\text { coordinated } \\
\text { movements of } \\
\text { upper and lower } \\
\text { limbs; trunk } \\
\text { movements in three } \\
\text { planes }\end{array}$ & $\begin{array}{l}\text { Reaction time; } \\
\text { visuospatial } \\
\text { attention, } \\
\text { shifting of } \\
\text { attention, } \\
\text { decision } \\
\text { making, } \\
\text { immediate } \\
\text { planning and } \\
\text { execution }\end{array}$ & MoCA & Not reported & $\begin{array}{l}\text { conventional } \\
\text { physiotherapy } \\
\text { including } \\
\text { balance, } \\
\text { endurance and } \\
\text { muscle } \\
\text { strength, } \\
\text { motor } \\
\text { coordination; } \\
\text { stretching }\end{array}$ & $\begin{array}{l}\text { Both groups } \\
\text { increased within all } \\
\text { performance } \\
\text { measurements; the } \\
\text { control group } \\
\text { increased walking } \\
\text { capacity }\end{array}$ \\
\hline Bisson [44] & $\begin{array}{l}\text { Specific } \\
\text { Virtual } \\
\text { reality DT } \\
\text { training }\end{array}$ & $\begin{array}{l}\text { Jiggle a virtual ball } \\
\text { while standing }\end{array}$ & $\begin{array}{l}\text { Reaction time; } \\
\text { visuospatial } \\
\text { attention, } \\
\text { immediate } \\
\text { planning and } \\
\text { execution }\end{array}$ & Reaction Time test & Not reported & $\begin{array}{l}\text { Biofeedback } \\
\text { training with } \\
\text { shifting the } \\
\text { center of mass }\end{array}$ & $\begin{array}{l}\text { No significant } \\
\text { group differences; } \\
\text { both groups } \\
\text { improved in the } \\
\text { cognitive task }\end{array}$ \\
\hline Chuang [45] & $\begin{array}{l}\text { GDT-EX - } \\
\text { video } \\
\text { dance }\end{array}$ & $\begin{array}{l}\text { Stepping forward, } \\
\text { backwards and } \\
\text { sidewards according } \\
\text { to the music and } \\
\text { presented steps on } \\
\text { a screen (following } \\
\text { an arrow) }\end{array}$ & $\begin{array}{l}\text { Reaction time, } \\
\text { Attention and } \\
\text { visuo-spatial } \\
\text { orientation }\end{array}$ & Flanker task & Not reported & $\begin{array}{l}\text { CG 1: brisk } \\
\text { walking } \\
\text { CG 2: inactive }\end{array}$ & $\begin{array}{l}\text { Reaction times } \\
\text { decreased in the } \\
\text { intervention group } \\
\text { as well as in the } \\
\text { brisk walking group }\end{array}$ \\
\hline $\begin{array}{l}\text { Eggenberger } \\
2015[27]\end{array}$ & $\begin{array}{l}\text { GDT-EX - } \\
\text { video } \\
\text { dance }\end{array}$ & $\begin{array}{l}\text { Stepping forward, } \\
\text { backwards and } \\
\text { sidewards according } \\
\text { to the music and } \\
\text { presented steps on } \\
\text { a screen (following } \\
\text { an arrow) }\end{array}$ & $\begin{array}{l}\text { Attention; } \\
\text { reaction time } \\
\text { and visuo- } \\
\text { spatial } \\
\text { orientation }\end{array}$ & $\begin{array}{l}\text { EF: Trail Making B } \\
\text { Long-term visual } \\
\text { memory, } \\
\text { Long-term verbal } \\
\text { memory (story } \\
\text { recall) } \\
\text { Wechsler Memory } \\
\text { scale revised }\end{array}$ & $\begin{array}{l}\text { Progression } \\
\text { adapted to } \\
\text { participants abilities }\end{array}$ & $\begin{array}{l}\text { CG1: treadmill } \\
\text { walking } \\
\text { memory } \\
\text { CG2: walking }\end{array}$ & $\begin{array}{l}\text { Both DT training } \\
\text { groups improved } \\
\text { the TMT-B; over a } \\
\text { longer period of } \\
\text { time the Dance } \\
\text { group still im- } \\
\text { proved whereas } \\
\text { the memory group } \\
\text { declined; same re- } \\
\text { sults for the execu- } \\
\text { tive control tasks; } \\
\text { GDT-EX improved } \\
\text { Working memory, } \\
\text { attentional control; } \\
\text { Go/no-go and set } \\
\text { shifting }\end{array}$ \\
\hline
\end{tabular}


Table 3 Data extraction of included studies (Continued)

\begin{tabular}{|c|c|c|c|c|c|c|c|}
\hline Author & $\begin{array}{l}\text { Training } \\
\text { intervention }\end{array}$ & Motor-component & $\begin{array}{l}\text { Cognitive } \\
\text { component }\end{array}$ & $\begin{array}{l}\text { Cognitive } \\
\text { measurement }\end{array}$ & Progression & $\begin{array}{l}\text { Control } \\
\text { group(s) }\end{array}$ & Results \\
\hline $\begin{array}{l}\text { Eggenberger } \\
2016 \text { [33] }\end{array}$ & $\begin{array}{l}\text { GDT-EX - } \\
\text { video } \\
\text { dance }\end{array}$ & $\begin{array}{l}\text { Stepping forward, } \\
\text { backwards and } \\
\text { sidewards according } \\
\text { to the music and } \\
\text { presented steps on } \\
\text { a screen (following } \\
\text { an arrow) }\end{array}$ & $\begin{array}{l}\text { Attention; } \\
\text { reaction time } \\
\text { and visuo- } \\
\text { spatial } \\
\text { orientation }\end{array}$ & $\begin{array}{l}\text { EF: Trail Making B } \\
\text { Stroop task } \\
\text { Working memory } \\
\text { task } \\
\text { MoCa } \\
\text { Processing speed }\end{array}$ & $\begin{array}{l}\text { Progression } \\
\text { adapted to } \\
\text { participants abilities }\end{array}$ & $\begin{array}{l}\text { Balance } \\
\text { training on } \\
\text { different } \\
\text { surfaces }\end{array}$ & $\begin{array}{l}\text { The intervention } \\
\text { group improved } \\
\text { the Trail making B, } \\
\text { MoCA and the } \\
\text { Stroop task }\end{array}$ \\
\hline Falbo [15] & GDT & $\begin{array}{l}\text { Physical- cognitive } \\
\text { DT training; walking } \\
\text { at different speeds; } \\
\text { coordination } \\
\text { training, balance } \\
\text { performance; } \\
\text { strengthening, } \\
\text { stretching with } \\
\text { music together with } \\
\text { different cognitive } \\
\text { tasks }\end{array}$ & $\begin{array}{l}\text { Inhibition, } \\
\text { working } \\
\text { memory and } \\
\text { set-shifting }\end{array}$ & $\begin{array}{l}\text { Random number } \\
\text { generation task to } \\
\text { address EF; dual } \\
\text { task cost while } \\
\text { walking }\end{array}$ & $\begin{array}{l}\text { Rising difficulties } \\
\text { (not further } \\
\text { described) }\end{array}$ & $\begin{array}{l}\text { Same exercise } \\
\text { program under } \\
\text { single task } \\
\text { condition }\end{array}$ & $\begin{array}{l}\text { The GDT group } \\
\text { improved cognitive } \\
\text { function }\end{array}$ \\
\hline Hars [34] & $\begin{array}{l}\text { GDT with } \\
\text { music }\end{array}$ & $\begin{array}{l}\text { Walking and } \\
\text { handling of objects; } \\
\text { reaction to the } \\
\text { rhythm of the music }\end{array}$ & Reaction time & $\begin{array}{l}\text { MMSE; Frontal } \\
\text { assessment battery } \\
\text { (FAB) }\end{array}$ & $\begin{array}{l}\text { Progression } \\
\text { mentioned but not } \\
\text { further described }\end{array}$ & $\begin{array}{l}\text { No } \\
\text { intervention }\end{array}$ & $\begin{array}{l}\text { Intervention group } \\
\text { increased MMSE }\end{array}$ \\
\hline Heiden [46] & $\begin{array}{l}\text { SDT } \\
\text { balance }\end{array}$ & $\begin{array}{l}\text { Body shifting to } \\
\text { control virtual } \\
\text { paddle }\end{array}$ & $\begin{array}{l}\text { Reaction time; } \\
\text { visuospatial } \\
\text { attention, } \\
\text { immediate } \\
\text { planning and } \\
\text { execution }\end{array}$ & Reaction time & $\begin{array}{l}\text { Chair based } \\
\text { exercise with } \\
\text { muscle } \\
\text { strengthening }\end{array}$ & $\begin{array}{l}\text { Reaction time } \\
\text { decreased in } \\
\text { the } \\
\text { intervention } \\
\text { group }\end{array}$ & \\
\hline Hiyamizu [35] & $\begin{array}{l}\text { GDT } \\
\text { balance }\end{array}$ & $\begin{array}{l}\text { Strength training, } \\
\text { balance and walking } \\
\text { training using } \\
\text { different } \\
\text { undergrounds in } \\
\text { combination with } \\
\text { verbal fluency, } \\
\text { arithmetic and } \\
\text { visual search task }\end{array}$ & $\begin{array}{l}\text { Working } \\
\text { memory } \\
\text { Visuospatial } \\
\text { tasks }\end{array}$ & $\begin{array}{l}\text { Trail making A and } \\
\text { B } \\
\text { Stroop task }\end{array}$ & Not reported & $\begin{array}{l}\text { Same program } \\
\text { but ST }\end{array}$ & $\begin{array}{l}\text { Only Stroop task } \\
\text { performance } \\
\text { improved in the } \\
\text { GDT group }\end{array}$ \\
\hline Kayama [28] & $\begin{array}{l}\text { GDT and } \\
\text { SDT } \\
\text { (exercises } \\
\text { with } \\
\text { specific } \\
\text { Dual task } \\
\text { Tai Chi) }\end{array}$ & $\begin{array}{l}\text { Aerobic training, } \\
\text { progressive muscle } \\
\text { strengthening, } \\
\text { flexibility and } \\
\text { balance; rhythmic } \\
\text { stepping exercise } \\
\text { with cognition; } 5 \\
\text { min Dual task Tai } \\
\text { Chi at the end }\end{array}$ & $\begin{array}{l}\text { Unclear; Dual } \\
\text { task Tai Chi } \\
\text { includes } \\
\text { visuospatial } \\
\text { tasks }\end{array}$ & $\begin{array}{l}\text { Verbal fluency test; } \\
\text { Trail making B }\end{array}$ & $\begin{array}{l}\text { Only reported for } \\
\text { strengthening }\end{array}$ & $\begin{array}{l}\text { Same training } \\
\text { than } \\
\text { intervention } \\
\text { group without } \\
\text { Dual task Tai } \\
\text { Chi }\end{array}$ & $\begin{array}{l}\text { the intervention } \\
\text { group improved } \\
\text { the Delta TMT }\end{array}$ \\
\hline Kitazewa [36] & $\begin{array}{l}\text { SDT net } \\
\text { step } \\
\text { exercises }\end{array}$ & $\begin{array}{l}\text { Steps within a net } \\
\text { in a predefined way; } \\
\text { every session } \\
\text { learning a new } \\
\text { combination; avoid } \\
\text { to step on the net; } \\
\text { than performing a } \\
\text { line with steps in } \\
\text { the net while singing } \\
\text { a children song }\end{array}$ & $\begin{array}{l}\text { Working } \\
\text { memory task }\end{array}$ & $\begin{array}{l}\text { Touch panel type } \\
\text { dementia scale; } \\
\text { Touch M system } \\
\text { addresses } \\
\text { visuospatial } \\
\text { function; the TDAS } \\
\text { is a modification of } \\
\text { the Alzheimer's } \\
\text { Desease } \\
\text { Asssessment Scale }\end{array}$ & $\begin{array}{l}\text { Increasing of steps } \\
\text { and difficulty of the } \\
\text { combination }\end{array}$ & $\begin{array}{l}\text { No } \\
\text { intervention }\end{array}$ & $\begin{array}{l}\text { Thouch M score } \\
\text { increased more in } \\
\text { the intervention } \\
\text { group; } \\
\text { Naming fingers as } \\
\text { part of the TDAS } \\
\text { improved in the } \\
\text { intervention group }\end{array}$ \\
\hline MacLean [37] & SDT & $\begin{array}{l}\text { Walking with } \\
\text { adjusting to the } \\
\text { speed of music; ST } \\
\text { walking, music } \\
\text { walking; DT walking } \\
\text { with music and } \\
\text { counting backwards }\end{array}$ & $\begin{array}{l}\text { Working } \\
\text { memory }\end{array}$ & $\begin{array}{l}\text { MMSE; TMT A-B; } \\
\text { Wechsler memory } \\
\text { scale revised } \\
\text { Digit span forward } \\
\text { and backward; Story } \\
\text { recall } \\
\text { DT walking }\end{array}$ & Not reported & $\begin{array}{l}\text { CG1: walking } \\
\text { to music } \\
\text { without } \\
\text { adjusting } \\
\text { CG2: walking } \\
\text { without music }\end{array}$ & $\begin{array}{l}\text { MT training } \\
\text { improved DT } \\
\text { walking }\end{array}$ \\
\hline
\end{tabular}


Table 3 Data extraction of included studies (Continued)

\begin{tabular}{|c|c|c|c|c|c|c|c|}
\hline Author & $\begin{array}{l}\text { Training } \\
\text { intervention }\end{array}$ & Motor-component & $\begin{array}{l}\text { Cognitive } \\
\text { component }\end{array}$ & $\begin{array}{l}\text { Cognitive } \\
\text { measurement }\end{array}$ & Progression & $\begin{array}{l}\text { Control } \\
\text { group(s) }\end{array}$ & Results \\
\hline Maillot [29] & $\begin{array}{l}\text { GDT-EX } \\
\text { (Nintendo } \\
\text { Wii) }\end{array}$ & $\begin{array}{l}\text { Body shifting and } \\
\text { arm movements in } \\
\text { front of the screen } \\
\text { or on the Wii } \\
\text { balance board }\end{array}$ & $\begin{array}{l}\text { Visuo spatial } \\
\text { tasks } \\
\text { Processing } \\
\text { speed tasks }\end{array}$ & $\begin{array}{l}\text { TMT A-B, Stroop } \\
\text { test } \\
\text { Letter set tests } \\
\text { Matrix reasoning } \\
\text { test } \\
\text { Digit symbol } \\
\text { substation test } \\
\text { Spatial span test } \\
\text { Directional heading } \\
\text { test } \\
\text { Mental rotation test } \\
\text { Cancellation test } \\
\text { Number } \\
\text { comparison test } \\
\text { Reaction time test } \\
\text { Plate tapping test }\end{array}$ & Not reported & Non active & $\begin{array}{l}\text { the intervention } \\
\text { group improved in } \\
\text { all cognitive tasks } \\
\text { except of the visuo } \\
\text { spatial tasks }\end{array}$ \\
\hline Morita [47] & GDT & $\begin{array}{l}\text { Mental gymnastics } \\
\text { with complicated } \\
\text { finger movements; } \\
\text { resistance training } \\
\text { with DT, aerobic } \\
\text { exercises with } \\
\text { changing } \\
\text { movement } \\
\text { directions and DT; } \\
\text { flexibility exercises }\end{array}$ & $\begin{array}{l}\text { Working } \\
\text { memory; } \\
\text { reaction time }\end{array}$ & $\begin{array}{l}\text { Modified } \\
\text { minimental State } \\
\text { (3MS) } \\
\text { TMT with a touch } \\
\text { panel }\end{array}$ & Not reported & Not active & $\begin{array}{l}\text { Intervention group } \\
\text { maintained } \\
\text { cognitive status } \\
\text { whereas control } \\
\text { group decreased }\end{array}$ \\
\hline $\begin{array}{l}\text { Nischiguschi } \\
\text { [38] }\end{array}$ & GDT & $\begin{array}{l}\text { Stretching, muscle } \\
\text { strength, DT } \\
\text { categories (working } \\
\text { memory, reaction } \\
\text { time, visuospatial } \\
\text { tasks) }\end{array}$ & $\begin{array}{l}\text { Working } \\
\text { memory, } \\
\text { reaction time } \\
\text { Visuospatial } \\
\text { tasks }\end{array}$ & $\begin{array}{l}\text { MMSE; Wechsler } \\
\text { memory scale } \\
\text { revised } \\
\text { TMT A-B; N-Back }\end{array}$ & $\begin{array}{l}\text { Reported for } \\
\text { strength training } \\
\text { but no further } \\
\text { details }\end{array}$ & $\begin{array}{l}\text { No } \\
\text { intervention }\end{array}$ & $\begin{array}{l}\text { Intervention group } \\
\text { better results in } \\
\text { WMS-R and TMT }\end{array}$ \\
\hline Ordnung [30] & $\begin{array}{l}\text { GDT- EX X } \\
\text { box }^{\text {TM}} 360 \\
\text { Kinect }^{\text {TM }}\end{array}$ & $\begin{array}{l}\text { Whole-body } \\
\text { movements to } \\
\text { move an avatar on } \\
\text { screen }\end{array}$ & $\begin{array}{l}\text { Attention, } \\
\text { visuospatial } \\
\text { function, } \\
\text { reaction time } \\
\text { Shifting and } \\
\text { decision } \\
\text { making }\end{array}$ & $\begin{array}{l}\text { Attention while } \\
\text { being seated with } \\
\text { Test of Attentional } \\
\text { Performance; } \\
\text { Simple reaction } \\
\text { time/Alertness } \\
\text { while being seated } \\
\text { in front of a } \\
\text { computer: response } \\
\text { (finger pressing) to } \\
\text { a visual stimuli on } \\
\text { screen; Working } \\
\text { memory (seated) } \\
\text { with the n-back } \\
\text { task) }\end{array}$ & Not reported & $\begin{array}{l}\text { No } \\
\text { intervention }\end{array}$ & $\begin{array}{l}\text { No significant } \\
\text { improvement in } \\
\text { tested cognitive } \\
\text { functions, but } \\
\text { improvements in } \\
\text { fine motor skills of } \\
\text { the left hand }\end{array}$ \\
\hline Schaettin [39] & GDT-EX & $\begin{array}{l}\text { lower extremity } \\
\text { movements, } \\
\text { stepping according } \\
\text { to force platform }\end{array}$ & $\begin{array}{l}\text { Attention; } \\
\text { reaction time } \\
\text { and visuo- } \\
\text { spatial } \\
\text { orientation }\end{array}$ & $\begin{array}{l}\text { Attention, } \\
\text { inhibition, working } \\
\text { memory (Test for } \\
\text { attentional } \\
\text { performance), } \\
\text { Cognitive Function } \\
\text { (MMSE) }\end{array}$ & $\begin{array}{l}\text { Not reported } \\
\text { (warm up } 5 \text { min; } \\
\text { training } 20 \text { min, } \\
\text { cool down } 5 \text { min) }\end{array}$ & $\begin{array}{l}\text { CG: traditional } \\
\text { balance } \\
\text { training, static } \\
\text { and dynamic } \\
\text { exercises, open } \\
\text { eyes and } \\
\text { closed eyes }\end{array}$ & $\begin{array}{l}\text { Four EF's increased } \\
\text { in the EXG group } \\
\text { and one (shifting) } \\
\text { in the CG }\end{array}$ \\
\hline $\begin{array}{l}\text { Schoene } \\
2013 \text { [31] }\end{array}$ & $\begin{array}{l}\text { SDT-EX } \\
\text { Dance } \\
\text { training }\end{array}$ & $\begin{array}{l}\text { Standing, stepping, } \\
\text { weight shifting }\end{array}$ & $\begin{array}{l}\text { Attention; } \\
\text { reaction time } \\
\text { and visuo- } \\
\text { spatial } \\
\text { orientation }\end{array}$ & $\begin{array}{l}\text { Processing speed } \\
\text { (Choice stepping } \\
\text { reaction time; TMT } \\
\text { A), shifting (TMT B), } \\
\text { Dual-task costs } \\
\text { (TUG-cog) }\end{array}$ & $\begin{array}{l}\text { Frist session } \\
\text { supervised by an } \\
\text { instructor, follow- } \\
\text { up sessions individ- } \\
\text { ualized sessions in } \\
\text { homes }\end{array}$ & $\begin{array}{l}\text { CG: no } \\
\text { intervention }\end{array}$ & $\begin{array}{l}\text { Improvement in } \\
\text { step reaction and } \\
\text { movement times }\end{array}$ \\
\hline
\end{tabular}


Table 3 Data extraction of included studies (Continued)

\begin{tabular}{|c|c|c|c|c|c|c|c|}
\hline Author & $\begin{array}{l}\text { Training } \\
\text { intervention }\end{array}$ & Motor-component & $\begin{array}{l}\text { Cognitive } \\
\text { component }\end{array}$ & $\begin{array}{l}\text { Cognitive } \\
\text { measurement }\end{array}$ & Progression & $\begin{array}{l}\text { Control } \\
\text { group(s) }\end{array}$ & Results \\
\hline $\begin{array}{l}\text { Schoene } \\
2015 \text { [40] }\end{array}$ & $\begin{array}{l}\text { SDT-EX } \\
\text { Dance } \\
\text { training }\end{array}$ & $\begin{array}{l}\text { Standing, stepping, } \\
\text { weight shifting }\end{array}$ & $\begin{array}{l}\text { Attention; } \\
\text { reaction time } \\
\text { and visuo- } \\
\text { spatial } \\
\text { orientation }\end{array}$ & $\begin{array}{l}\text { Inhibition (Stoop } \\
\text { Stepping Test); } \\
\text { Working Memory } \\
\text { (letter-digit test, } \\
\text { digit span } \\
\text { backwards), } \\
\text { Processing speed/ } \\
\text { Attention (Test for } \\
\text { attentional network } \\
\text { test, TMTA, CRT+ } \\
\text { CSRT; shifting (TMT } \\
\text { B); Dual task costs } \\
\text { (TUG-cog) }\end{array}$ & $\begin{array}{l}\text { Instruction at the } \\
\text { beginning of the } \\
\text { trial; conduction } \\
\text { unsupervised in } \\
\text { individuals' homes }\end{array}$ & $\begin{array}{l}\text { CG: } \\
\text { educational } \\
\text { brochure in } \\
\text { falls prevention }\end{array}$ & $\begin{array}{l}\text { IG improvement in } \\
\text { processing speed } \\
\text { and mental } \\
\text { rotation, set- } \\
\text { shifting increased } \\
\text { with a higher dose } \\
\text { of game playing; } \\
\text { individuals with } \\
\text { poorer baseline } \\
\text { function in IG } \\
\text { showed greater } \\
\text { improvement }\end{array}$ \\
\hline Theill [48] & GDT & $\begin{array}{l}\text { Cardiovascular } \\
\text { treadmill training; } \\
\text { walking }\end{array}$ & $\begin{array}{l}\text { Verbal Working } \\
\text { memory }\end{array}$ & $\begin{array}{l}\text { (selective) Attention, } \\
\text { working memory, } \\
\text { paired associates } \\
\text { learning, processing } \\
\text { speed, Dual task } \\
\text { costs }\end{array}$ & Not reported & $\begin{array}{l}\text { IG2: working } \\
\text { memory } \\
\text { training } \\
\text { (single) } \\
\text { CG: no } \\
\text { intervention }\end{array}$ & $\begin{array}{l}\text { Improvement in } \\
\text { executive control, } \\
\text { no improvement in } \\
\text { selective attention, } \\
\text { more improvement } \\
\text { in IG in paired } \\
\text { associates learning }\end{array}$ \\
\hline $\begin{array}{l}\text { Wollesen } \\
\text { 2017a [41] }\end{array}$ & GDT & $\begin{array}{l}\text { Standing, balancing, } \\
\text { muscle training }\end{array}$ & $\begin{array}{l}\text { Working } \\
\text { memory, } \\
\text { reaction time } \\
\text { Visuospatial } \\
\text { tasks; task } \\
\text { prioritization, } \\
\text { task shifting }\end{array}$ & $\begin{array}{l}\text { Dual tasks costs } \\
\text { (walking under DT } \\
\text { and ST conditions), } \\
\text { Inhibition (seated } \\
\text { Stroop Test/ } \\
\text { walking while } \\
\text { undertaking Stroop } \\
\text { Test) }\end{array}$ & $\begin{array}{l}\text { Two phase } \\
\text { intervention: Phase } \\
1 \text { (wk. 1-6); training } \\
\text { of daily actions } \\
\text { with likelihood of } \\
\text { fall risks; Phase } 2 \\
\text { (wk. 7-12) Task } \\
\text { priorization }\end{array}$ & $\begin{array}{l}\text { IG2 single task } \\
\text { strength and } \\
\text { resistance } \\
\text { CG: no } \\
\text { intervention }\end{array}$ & $\begin{array}{l}\text { No significant } \\
\text { improvement in } \\
\text { IG1 in cognitive } \\
\text { functions }\end{array}$ \\
\hline $\begin{array}{l}\text { Wollesen } \\
\text { 2017b [42] }\end{array}$ & GDT & $\begin{array}{l}\text { Walking, standing, } \\
\text { balancing, }\end{array}$ & $\begin{array}{l}\text { Working } \\
\text { memory, } \\
\text { reaction time } \\
\text { Visuospatial } \\
\text { tasks; task } \\
\text { prioritization, } \\
\text { task shifting }\end{array}$ & $\begin{array}{l}\text { Dual-task costs } \\
\text { (walking under ST } \\
\text { and DT conditions), } \\
\text { Inhibition (verbal } \\
\text { Stroop task) }\end{array}$ & $\begin{array}{l}\text { Two phase } \\
\text { intervention: Phase } \\
1 \text { (wk. 1-6); training } \\
\text { of daily actions } \\
\text { with likelihood of } \\
\text { fall risks; Phase } 2 \\
\text { (wk. 7-12) Task } \\
\text { priorization and } \\
\text { transfer into daily } \\
\text { life }\end{array}$ & $\begin{array}{l}\text { IG2: ST } \\
\text { conditions } \\
\text { CG: no } \\
\text { intervention }\end{array}$ & $\begin{array}{l}\text { Reduced number } \\
\text { of errors in IG in } \\
\text { Stroop test }\end{array}$ \\
\hline You [43] & SDT & Walking, standing & Memorizing & $\begin{array}{l}\text { Working memory } \\
\text { (memory recall \%) }\end{array}$ & Not reported & $\begin{array}{l}\text { CG: no } \\
\text { intervention }\end{array}$ & $\begin{array}{l}\text { Memory } \\
\text { performance } \\
\text { improved under DT } \\
\text { conditions }\end{array}$ \\
\hline
\end{tabular}

walking exercise combined with an alphabetical task while testing verbal fluency. Eggenberger and colleagues [33] and Schoene and colleagues [31, 40] used a dancing exergame for theirs trials, which required the participant to take a step in different directions following instructions on the screen, while controlling speed and inhibiting a step presented in a different colour. Wollesen and colleagues $[41,42]$ conducted a progressive task-managing training (e.g. task prioritisation and task switching) in combination with balance and walking exercises. These trials used SDT and GDT interventions and had a duration between twelve and 36 h. The remaining five studies showed no significant change in inhibitory control $[15,26,30,39,45]$. These trials used SDT and GDT interventions and had a duration between twelve and $24 \mathrm{~h}$. However, the studies differed with regard to the activities of the control groups (cf. Table 3). A meta-analysis was conducted on seven of these studies.

As shown in Fig. 3, studies with an active control group showed benefits on inhibitory control with a significant mean difference of 0.71 [95\% CI 0.33-1.09], whereas the studies with inactive controls did not. However, heterogeneity was high with $\mathrm{I}^{2}=95 \%$. This large heterogeneity could have been a result of the data by Eggenberger and colleagues [33]. After running a sensitivity analysis without the study by Eggenberger and colleagues [33] the heterogeneity reduced to $\mathrm{I}^{2}=46 \%$ and the effect between the training and the control groups did not remain significant. 


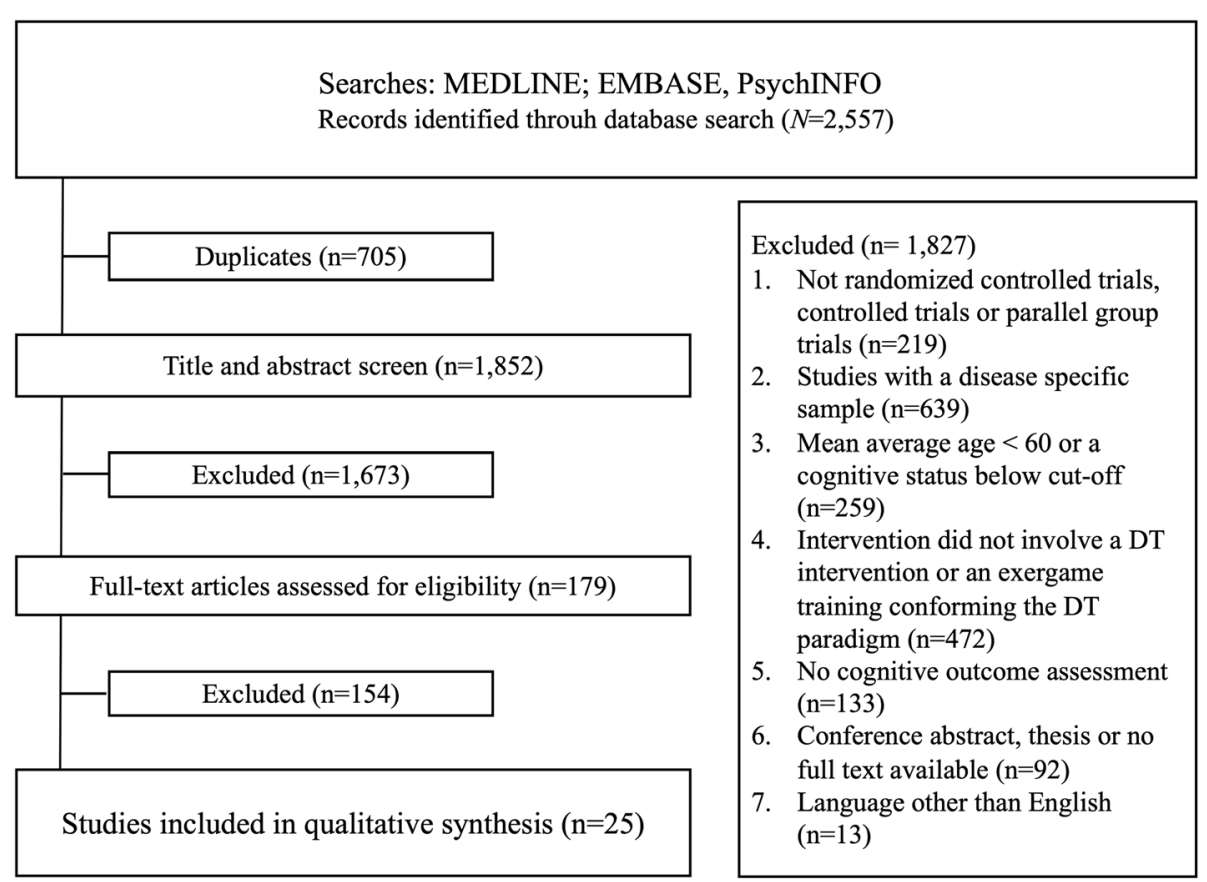

Fig. 1 Flow chart of study selection process

\section{Effects on working memory}

Seven out of nine interventions reported improved working memory $[26,27,30,38,40,43,48]$. Three of these interventions were GDT exergames [27, 30, 40] three were GDT programs [26, 38, 43, 48] and You and colleagues [43] provided a DT walking training with additional working memory tasks. The interventions conducted by Falbo and colleagues [15] and MacLean and colleagues [37] did not increase the examined working memory task.

The results of the meta-analysis for working memory showed a significant mean difference of 2.09 [95\% CI -0.1-4.30] of the two studies who had an inactive control group. The comparison of a DT intervention with physical active controls did not show advantages for the DT training on improving working memory. In addition, there was no overall effect on working memory for the integrated five studies in the meta-analysis (cf. Fig. 4). Heterogeneity was moderate (I $2=47 \%)$, mainly caused by the studies with an active control group (I2 =57\%).

\section{Effects on cognitive flexibility (i.e. set-shifting)}

Seven out of ten interventions showed improved set-shifting after a GDT or a GDT exergame intervention, using the TMT A/B, TMT B-A or the verbal random number generation test (RNG) [15, 27, 29, 33, 35, 38, 40]. The training duration of these interventions was between twelve and 36 $\mathrm{h}$. The three interventions by Kayama and colleagues [28] (a GDT in combination with SDT Tai Chi), MacLean and colleagues [37] (SDT with walking to music) and Schaettin and colleagues [39] (GDT exergame with stepping on a force platform) did not increase set-shifting abilities. The metaanalysis of five studies that could be added into the analysis did not show any effects of the DT interventions (cf. Fig. 5).

\section{Effects on visuospatial planning}

Four studies intended to improve visuospatial planning by using GDT $[25,29,36,40]$. Only the programs run by Kitazawa and colleagues [36] and Schoene and colleagues [40] improved visuospatial planning.

\section{Effects on attentional control}

Attentional control was examined in nine of the training studies. Seven of these led to significant improvements of attentional control. Four of these studies used a GDT intervention [35, 38, 41, 42, 47]. Two studies conducted a SDT exergame intervention $[39,40]$ and one used a GDT exergame [27].

The GDT programs by Ansai and colleagues [25] and Theill and colleagues [48] failed to show effects on attentional control. Both studies conducted a GDT program, which required the participants to engage in walking as well as resistance training interventions combined with a numerical task or a cognitive load.

\section{Effects on processing speed}

Processing speed was the most examined variable within the cognitive tasks of the included studies. A total number of 15 studies focused on this outcome. All 
Table 4 Data extraction - Results on different cognitive dimensions

\begin{tabular}{|c|c|c|c|c|c|c|c|c|c|c|}
\hline Author Year & $\begin{array}{l}\text { GDT / SDT } \\
\text { / EXG }\end{array}$ & $\begin{array}{l}\text { Global cognitive } \\
\text { function }\end{array}$ & $\begin{array}{l}\text { set- } \\
\text { shifting }\end{array}$ & $\begin{array}{l}\text { inhibitory } \\
\text { control }\end{array}$ & $\begin{array}{l}\text { working } \\
\text { memory }\end{array}$ & $\begin{array}{l}\text { visuospatial } \\
\text { planning }\end{array}$ & $\begin{array}{l}\text { verbal } \\
\text { fluency }\end{array}$ & $\begin{array}{l}\text { attentional } \\
\text { control }\end{array}$ & $\begin{array}{l}\text { processing } \\
\text { speed }\end{array}$ & $\begin{array}{l}\text { dual-task } \\
\text { costs }\end{array}$ \\
\hline Ansai [25] & GDT & n.s & n.a. & n.a. & n.a & n.s. & n.a & n.s & n.a & $\downarrow$ \\
\hline Azadian [26] & GDT & n.a. & n.a. & $\begin{array}{l}|\mathrm{GG} 2: \uparrow ;| \mathrm{G} 1: \\
\text { n.s. }\end{array}$ & IGI: $\uparrow$ & n.a. & n.a. & n.a. & n.s. & n.a. \\
\hline Bacha [32] & GDT- EXG & $\mathrm{IG}+\mathrm{CG} \uparrow$ & n.a. & n.a. & n.a. & n.a & n.a.. & n.a. & n.a.. & n.a. \\
\hline Bisson [44] & GDT- EXG & n.a. & n.a. & n.a. & n.a & n.a. & n.a & n.a. & $\mid \mathrm{G} 1+2 \uparrow$ & n.a. \\
\hline Chuang [45] & SDT- EXG & n.a. & n.a. & n.s. & n.a & n.a. & n.a & n.a. & $\begin{array}{l}\text { RT IG: } \uparrow R T \\
\text { CG: } \downarrow\end{array}$ & n.a \\
\hline $\begin{array}{l}\text { Eggenberger } \\
2015 \text { [27] }\end{array}$ & GDT -EXG & n.a. & $\begin{array}{l}I G 1+I G \\
2: \uparrow\end{array}$ & n.a. & $\begin{array}{l}\text { IG } 1+\mid \mathrm{G} 2: \\
\uparrow\end{array}$ & n.a. & n.a & IG $1+\mid \mathrm{G} 2: \uparrow$ & $\uparrow$ & n.a \\
\hline $\begin{array}{l}\text { Eggenberger } \\
2016 \text { [33] }\end{array}$ & GDT- EXG & n.s & $\uparrow$ & n.a. & n.a & n.a. & n.a & n.a. & $\uparrow$ & n.a \\
\hline Falbo [15] & GDT & n.a. & $\begin{array}{l}\mathrm{IG}: \uparrow \\
\mathrm{CG} 1: \downarrow\end{array}$ & n.s. & n.s. & n.a. & n.a & n.a. & n.a & n.a \\
\hline Hars [34] & GDT & $\uparrow$ & n.a. & n.a. & n.a & n.a. & n.a. & n.a. & n.a & n.a. \\
\hline Heiden [46] & GDT & n.a. & n.a & n.a. & n.a & n.a. & n.a & n.a. & IG: $\uparrow$ & n.a. \\
\hline Hiyamizu [35] & GDT & n.a. & n.s. & IG: $\uparrow$ & n.a. & n.a. & n.a. & n.a. & n.s. & n.a. \\
\hline Kayama [28] & $\mathrm{SDT}+\mathrm{EXG}$ & n.a. & n.a. & n.a. & n.a. & n.a. & n.s. & n.a. & n.s. & n.a. \\
\hline Kitazawa [36] & GDT & IG: $\uparrow$ & n.a. & n.a. & n.a. & IG: $\uparrow$ & n.a. & n.a. & n.a. & n.a. \\
\hline MacLean [37] & GDT & n.a. & n.s. & n.a. & n.s. & n.a. & n.a. & n.a. & n.s. & n.a. \\
\hline Malliot [29] & GDT-EXG & n.a. & IG: $\uparrow$ & IG: $\uparrow$ & IG: $\uparrow$ & n.s. & n.a. & n.a. & IG: $\uparrow$ & n.a. \\
\hline Morita [47] & GDT & n.s. & n.s.. & n.a. & n.a. & n.a. & n.s. & n.s. & n.a. & n.a \\
\hline Nishiguchi [38] & GDT & n.s. & IG: $\uparrow$ & n.a. & IG: $\uparrow$ & n.a. & n.a. & IG: $\uparrow$ & IG: $\uparrow$ & n.a. \\
\hline Ordnung [30] & GDT- EXG & n.a. & n.a. & n.s. & n.s. & n.a. & n.a. & n.a. & n.s. & n.a. \\
\hline $\begin{array}{l}\text { Schaettin } 2016 \\
\text { [39] }\end{array}$ & SDT- EXG & n.a. & IG: $\uparrow$ & IG: $\uparrow$ & IG: $\uparrow$ & n.a. & n.a. & IG: $\uparrow$ & n.a. & n.a. \\
\hline $\begin{array}{l}\text { Schoene } 2013 \\
\text { [31] }\end{array}$ & SDT- EXG & n.a. & n.a. & IG: $\uparrow$ & n.a. & n.a. & n.a. & n.a. & IG: $\uparrow$ & n.a. \\
\hline $\begin{array}{l}\text { Schoene } 2015 \\
\text { [40] }\end{array}$ & SDT-EXG & n.a. & IG: $\uparrow$ & n.s. & IG: $\uparrow$ & n.a. & n.a. & $\uparrow$ & IG: $\uparrow$ & n.a. \\
\hline Theill [48] & GDT & n.a & n.a. & n.a. & IG $1: \uparrow$ & n.a. & n.a. & n.s. & n.a. & n.a. \\
\hline $\begin{array}{l}\text { Wollesen } \\
\text { 2017a [41] }\end{array}$ & GDT & n.a. & n.a. & n.s. & n.a. & n.a. & n.a. & n.s. & n.a. & $\downarrow$ \\
\hline $\begin{array}{l}\text { Wollesen } \\
\text { 2017b [42] }\end{array}$ & GDT & n.a. & n.a. & IG: $\uparrow$ & n.a. & n.a. & n.a. & $\uparrow$ & n.a. & $\downarrow$ \\
\hline You [43] & SDT & n.a. & n.a. & n.a. & IG: $\uparrow$ & n.a. & n.a. & n.a. & n.a. & n.a. \\
\hline
\end{tabular}

IG Intervention Group, CG Control Group, GDT General dual-tasking, SDT Specific dual-tasking, EXG Exergame, $\uparrow$ - performance increase, $\downarrow$ - performance decrease, n.s. non significant, n.a. non available, RT Reaction time, TMT Trail Marking Test, $\Delta-$ delta score

exergaming interventions $(n=8)$ except of the one by Kayama and colleagues [28] showed improvement of processing speed [27, 29-31, 33, 40, 44, 45]. Moreover, the two GDT programs by Heiden \& Lajoie [46], and Nishiguschi and colleagues [38] improved processing speed.

In contrast the GDT programs by Azadian and colleagues [26], Hiyamizu and colleagues [35], MacLean and colleagues [37] and Morita and colleagues [47] did not affect processing speed. All trials used a GDT walking intervention, which was combined with different cognitive tasks, e.g. counting backwards or naming out of memory. MacLean and colleagues [37] and Hars and colleagues [34] both engaged their participants in a pace adaption task, which required participants to adjust the speed of their pace to the rhythm of the music played along the walking exercise.

\section{Effects on DTC}

The studies by Ansai and colleagues [25]; MacLean and colleagues [37] and Wollesen and colleagues [42] also addressed cognitive-motor interference by analysing 


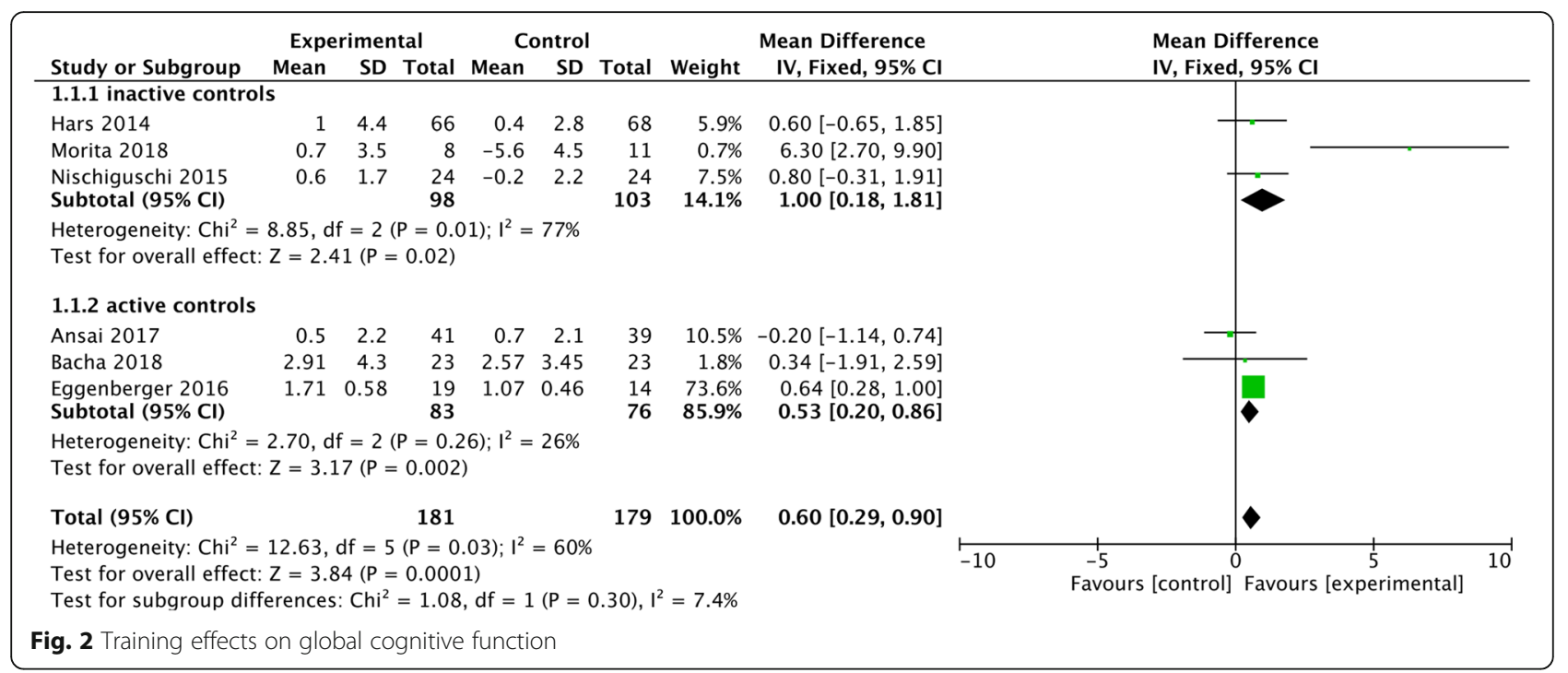

DTCs. All programs were able to reduce cognitivemotor interference, leading to improved performance on the cognitive or motor task.

\section{Influence of training methods}

Overall, 24 interventions were able to improve at least one cognitive outcome. The majority of the studies increased the cognitive outcome that was specific to the training intervention (cf. Table 3). Three studies were not able to show effects on their cognitive test battery $[25,30,47]$. The studies conducted by Ansai and colleagues [25] and Morita and colleagues [47] integrated a cognitive test that was not correlated to the cognitive dimensions that were addressed in the training program.

Moreover, seven studies showed improvement of cognitive abilities that were specifically addressed as a part of the training intervention. Three of them were exergaming interventions $[27,33,40]$. In addition, the nine exergaming studies that examined effects on processing speed or reaction times were all able to show improvements on processing speed.

A progression in ST or DT complexity was provided by nine interventions (cf. Table 3). Four studies used an individualised adaption to the training difficulties (cf. Table 3). All eight studies with an individualised or progressive training that examined executive functions

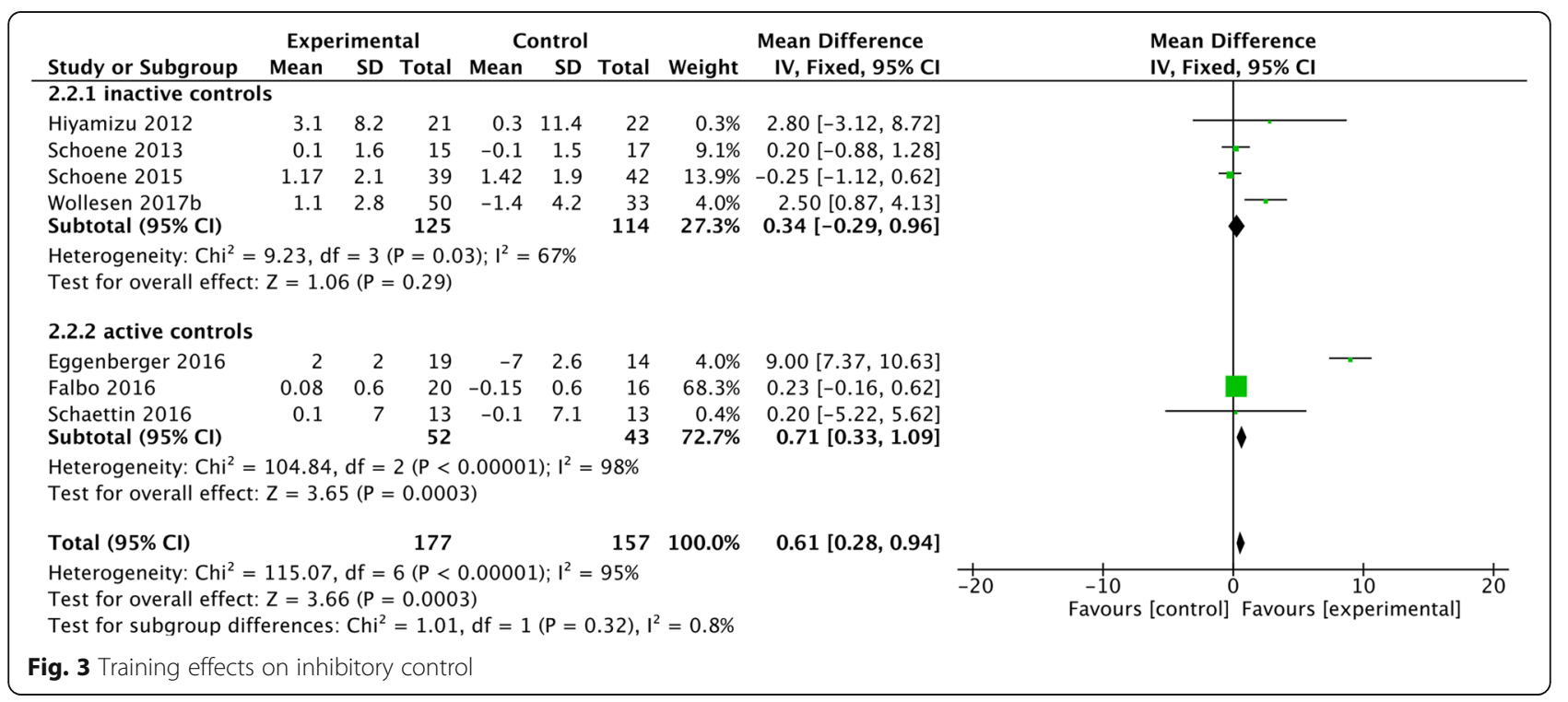




\begin{tabular}{|c|c|c|c|c|c|c|c|c|c|c|c|c|}
\hline \multirow[b]{2}{*}{ Study or Subgroup } & \multicolumn{3}{|c|}{ Experimental } & \multicolumn{3}{|c|}{ Control } & \multicolumn{3}{|c|}{ Mean Difference } & \multirow{2}{*}{\multicolumn{2}{|c|}{$\begin{array}{l}\text { Mean Difference } \\
\text { IV, Fixed, } 95 \% \mathrm{CI}\end{array}$}} & \\
\hline & Mean & SD & Total & Mean & SD & Total & Weight & IV, Fixed, 95\% CI & & & & \\
\hline \multicolumn{13}{|c|}{ 3.1.1 inactive controls } \\
\hline Nischiguschi 2015 & 4.7 & 8.2 & 24 & 1.3 & 7.7 & 24 & $1.8 \%$ & $3.40[-1.10,7.90]$ & & & 5 & \\
\hline $\begin{array}{l}\text { Schoene } 2015 \\
\text { Subtotal }(95 \% \mathrm{CI})\end{array}$ & 2.5 & 5.9 & $\begin{array}{l}39 \\
63\end{array}$ & 0.82 & 5.7 & $\begin{array}{l}42 \\
66\end{array}$ & $\begin{array}{l}5.7 \% \\
7.5 \%\end{array}$ & $\begin{array}{l}1.68[-0.85,4.21] \\
2.09[-0.114 .30]\end{array}$ & & & 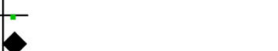 & \\
\hline \multicolumn{13}{|c|}{$\begin{array}{l}\text { Heterogeneity: } \mathrm{Chi}^{2}=0.43, \mathrm{df}=1(P=0.51) ; \mathrm{I}^{2}=0 \% \\
\text { Test for overall effect: } Z=1.86(P=0.06)\end{array}$} \\
\hline \multicolumn{13}{|l|}{ 3.1.2 active controls } \\
\hline You 2009 & 31.3 & 16.4 & 8 & 15 & 10.5 & 5 & $0.2 \%$ & $16.30[1.68,30.92]$ & & & & \\
\hline Schaettin 2016 & 2.1 & 14 & 13 & 0.6 & 13.8 & 14 & $0.3 \%$ & $1.50[-9.00,12.00]$ & & & & \\
\hline $\begin{array}{l}\text { Falbo } 2016 \\
\text { Subtotal }(95 \% \mathrm{Cl})\end{array}$ & 0.12 & 1.2 & $\begin{array}{l}20 \\
41\end{array}$ & -0.11 & 0.7 & $\begin{array}{l}16 \\
35\end{array}$ & $\begin{array}{l}92.0 \% \\
92.5 \%\end{array}$ & $\begin{array}{l}0.23[-0.40,0.86] \\
0.26[-0.36,0.89]\end{array}$ & & & 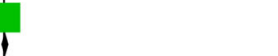 & \\
\hline \multicolumn{13}{|c|}{$\begin{array}{l}\text { Heterogeneity: } \mathrm{Chi}^{2}=4.68, \mathrm{df}=2(P=0.10) ; I^{2}=57 \% \\
\text { Test for overall effect: } Z=0.83(P=0.41)\end{array}$} \\
\hline Total $(95 \% \mathrm{Cl})$ & & & 104 & & & 101 & $100.0 \%$ & $0.40[-0.20,1.00]$ & & & & \\
\hline \multicolumn{9}{|c|}{$\begin{array}{l}\text { Heterogeneity: } \mathrm{Chi}^{2}=7.56, \mathrm{df}=4(\mathrm{P}=0.11) ; \mathrm{I}^{2}=47 \% \\
\text { Test for overall effect: } Z=1.30(P=0.19) \\
\text { Test for subgroup differences: } \mathrm{Chi}^{2}=2.45, \mathrm{df}=1(P=0.12), \mathrm{I}^{2}=59.1 \%\end{array}$} & -50 & $\begin{array}{c}-25 \\
\text { Favours [control] }\end{array}$ & $\begin{array}{l}25 \\
\text { Favours [experimental] }\end{array}$ & $\overrightarrow{50}$ \\
\hline Fig. 4 Training effects & on wo & rking $r$ & nemor & & & & & & & & & \\
\hline
\end{tabular}

were able to improve these functions. Cognitive-motor training was more effective in comparison to other training interventions if it combined cognitive training with multicomponent exercises including balance or coordination tasks (cf. Table 3).

\section{Influence of overall dose}

The frequency, session duration and dose of the interventions ranged from 1 to 3 days per week, 20 to $90 \mathrm{~min}$ per session and an overall dose of eight to $104 \mathrm{~h}$ in total. The shortest intervention duration was 4 weeks and the longest was 16 weeks. The frequency, session duration and dose of the interventions for studies with positive effects on one or more cognitive functions did not differ for studies not showing effects on cognitive function.
To improve global cognitive functioning the total training duration ranged between eight and $104 \mathrm{~h}$ [32, $34,36,38,47]$. The frequency differed between one or two times per week with 30, 60 or 90 min sessions.

Inhibitory control increased with interventions provided between 12 and $24 \mathrm{~h}$. They were conducted once [41, 42], twice [35] or three times per week [31, 33].

Effects on working memory was shown with training programs that provided 12 up to $36 \mathrm{~h}$ of exercise [26, 27, $30,38,40,43,48]$. The frequency differed between once a week [48], twice [30, 38], three times [26, 27, 40] and five sessions [43].

Benefits on set-shifting was gained by exercise interventions with a duration between 12 and $36 \mathrm{~h}$ with a frequency of one time per week $[29,38]$, twice per week $[15,35]$ or three times per week $[27,33,40]$.

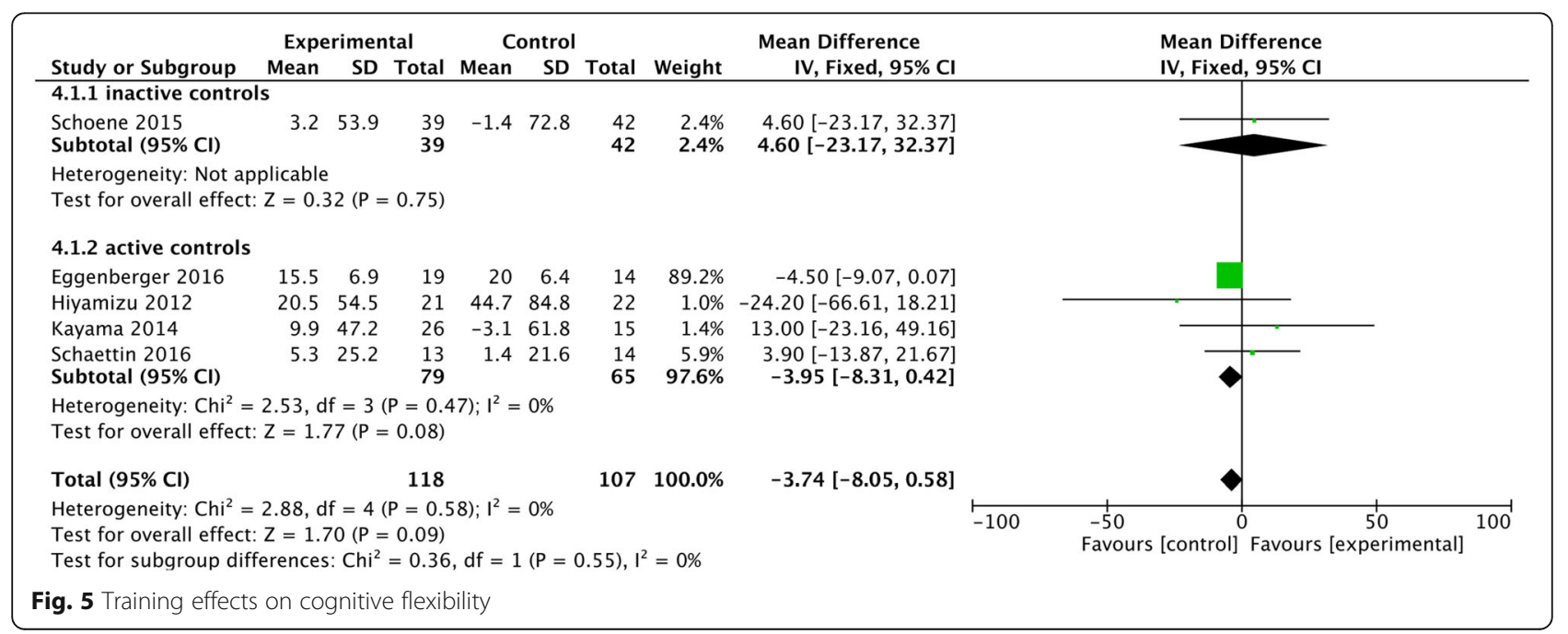


The programs that were able to improve visuospatial planning were provided once a week with $60 \mathrm{~min}$ for 7 weeks with a total duration of $8 \mathrm{~h}$ [36] and three times a week for $20 \mathrm{~min}$ with a total duration of $16 \mathrm{~h}$ [40].

Attention control increased with exercise interventions of a duration between 12 and $104 \mathrm{~h}$ of training [35, 38, $41,42,47]$. Four of these programs were executed once a week with 60 to $90 \mathrm{~min}[38,41,42,47]$. The intervention by Hiyamazu and colleagues had two 60 min sessions per week.

Improvements of processing speed were found for exercise programs with a duration of eight up to $36 \mathrm{~h}$. Most of these interventions were exergames that were provides three times per week for 20,30 or $60 \mathrm{~min}$ [27, $31,33,40,45]$. The other programs had a frequency of two times a week with 30 or $60 \mathrm{~min}[30,44,46]$ or once session per week with 60 or 90 min $[29,38]$.

Dual-task costs were reduced with interventions with a duration from twelve provided once a week for $60 \mathrm{~min}$ [42] or $60 \mathrm{~h}$ of training three times a week with $50 \mathrm{~min}$ [25]; MacLean and colleagues [37] did not report their training duration and frequency.

In summary, a dose of at least $60 \mathrm{~min}$ training per week (regardless of the number of sessions per week) and a total duration of $12 \mathrm{~h}$ of training was necessary to gain positive effects on the different cognitive domains.

\section{Discussion}

The aim of this review and meta-analysis was to investigate whether cognitive-motor interventions improve cognitive function in healthy older people. We focused on interventions that implemented conventional dualtask training and examined whether training methods show a beneficial effect on global cognition and specific executive functions (inhibitory control, working memory and cognitive flexibility). Secondly, we investigated cognitive effects of technology-based dual-task interventions, so called exergames. Overall, we found equal proportions of general cognitive-motor $(n=14)$ and exergame interventions $(n=11)$. Most studies conducted a GDT $(n=19)$, while we also found some studies using SDT $(n=6)$. However, the studies differed in experimental design, training type. Dose as well as outcome or control assessment.

While all studies used valid experimental designs, the training settings differed in many of the investigated trials. Moreover, sample sizes showed great differences. For example, You and colleagues [43], Morita and colleagues [47] and Heiden \& Lajoie [46] showed significant results for at least two specific executive functions in their population, with a sample size of less than 20 participants. In comparison, Eggenberger and colleagues [27] included more than 80 participants, which might have led to a higher statistical power and stronger result. These interpretations were supported by the additional sensitivity analysis. After excluding the studies of Morita and colleagues [47] and Eggenberger and colleagues [27] the heterogeneity was reduced, however, the significant results also disappeared. Additionally, the pre-assessment of cognitive ability of participants differed in a majority of studies. Many studies used a valid measurement, e.g. MMSE or MoCA scores, however diversity in testing instruments, e.g. Mini-Cog, 3MS or TDAS score, as well as diversity in the population's baseline characteristics hamper interstudy comparison. As the reason of this review is to summarise cognitive effects, the same mental baseline condition is of importance to allow a more detailed interpretation of results.

Despite the use of valid assessment instruments to quantify results across all studies, measurements were likewise heterogeneous, hindering a holistic interpretation of effects. For example, studies used different versions of established measurements, e.g. of the Stroop task, which could be delivered as an audioverbal, as well as visual-verbal or visual-manual task. In general, assessment instruments varied between written or computerised measurements to the active conduction of specific functions, which increased difficulty of comparing assessment structures.

\section{Effects on global cognition and executive functions}

We found that general cognitive-motor interventions improve global cognition and executive functions. All but one study found improvement of at least one specific function. Of note, type of exercise, dose, intervention settings and outcomes differed across trials which allowed for fractional interpretation of the given results only.

\section{Effects on global cognition}

Our meta-analysis of six studies revealed that cognitivemotor interventions improve global cognition. However, the effect size of this improvement was relatively small (standardised mean difference of 0.6 points on the MMSE and MoCA, 95\% CI 0.3-0.9) and the heterogeneity between studies was relatively large $\left(\mathrm{I}^{2}=60 \%\right)$, suggesting that additional evidence is required. Only two studies showed a statistically significant improvement, driving the results of the meta-analyses [27, 47]. Both studies were considered of high quality. The intervention of Morita and colleagues [47] comprised of a total of $104 \mathrm{~h}$ of conventional DT training, with 3MMS as their global cognition outcome, and the intervention of Eggenberger and colleagues [27] comprised of $18 \mathrm{~h}$ of DT video game training, with $\mathrm{MoCA}$ as their global cognition outcome. While the intervention of Eggenberger and colleagues [27] included task-specific exercises with 
individual adjusted progressions, the program of Morita and colleagues [47] had a training period of 18 months. This suggests that both training specificity and a long intervention period might be beneficial to improve cognitive function.

Nevertheless, it has to be reflected, that the MMSE and $\mathrm{MoCA}$ are assessment and screening tools, and not typically used as outcome measures. Therefore, further research is required to identify effective components of cognitive-motor interventions for improving global cognition with more sensitive test batteries (e.g. Mindsteams [49] or CERAD-col [50].

\section{Effects on inhibitory control}

Six out of eleven DT training programs significantly improved inhibitory control [31, 33, 35, 40-42]. The meta-analysis revealed that cognitive-motor interventions improved inhibitory control. The effect size was $\mathrm{Z}=3.66$ ( $p<0.001$; mean difference $0.61,95 \%$ CI $0.33-$ 1.09 ) with high heterogeneity $\left(\mathrm{I}^{2}=95 \%\right)$. Remarkably, this effect was strongest when comparing interventions to active controls, possibly the result of one study by Eggenberger and colleagues [33] with a very large effect size $\mathrm{Z}=3.85(p<0.001$; mean difference of $9.0,95 \% \mathrm{CI}$ 7.4-10.6). This study comprised of $18 \mathrm{~h}$ of DT video game, that included stepping forward, backwards and sideways to music while following cues (i.e. arrows) presented on a screen.

Most programs that showed benefits of inhibitory control included specific training of this cognitive ability in the intervention [31, 33, 41, 42]. The training duration to gain positive effects was at least $12 \mathrm{~h}$. This suggests ageing may affect executive and automatic inhibition differently [51]. Executive inhibition involves consciousness as well as control for the suppression of irrelevant stimuli or responses [51]. These abilities are integrated within an array of training interventions and physical exercise that integrate balance and coordination tasks, because these exercises require skills and cognitive effort to respond to immediate external stimuli that might arise from an unpredictable environment or situations [45]. Therefore, one might argue that these interventions are beneficial to improve executive functions, including inhibitory control. We further found that inhibitory control improved following cognitive-motor exercises that focus on attentional performance. This supports previous findings of improved attentional and inhibition performance following a dualtask training intervention $[8,52-54]$.

\section{Effects on working memory}

Working memory only improved in trials which specifically addressed memory abilities during training situations. Most studies used a memory task while conducting physical exercise, which appeared to be stimulating memory function. The results of the metaanalysis for working memory showed no significant effects. A previous review by Kelly and colleagues [55] was able to demonstrate that specific cognitive training can improve the addressed domains of cognition, whereas results of a general mental stimulation were heterogeneous. As indicated by the studies that improved working memory (cf. Table 3), there should be a certain amount of task specific training to gain positive effects on working memory. Accordingly, this might be the reason why the study data remained inconsistent if the task specificity was low.

\section{Effects on cognitive flexibility}

Set-shifting was analysed as a primary outcome in nine studies (cf. Table 3). Seven of the examined interventions showed improvement of set-shifting after a GDT or a GDT exergaming intervention. Unfortunately, only a few studies could be included in the meta-analysis, showing no significant effects of cognitive-motor interventions on set-shifting. However, set-shifting is an executive function that involves unconscious and conscious cognitive shifting of attention [10,15]. Such processes can be supported by processing speed. Most of the exergaming interventions $(n=9)$ were able to increase processing speed; future studies could elucidate whether these effects transfer to improvements in set-shifting ability.

\section{Influence of the training methods}

Based on the presented results no major differences in results between methods could be identified. A tendency towards a positive impact on processing speed and attentional control was observed within the exergaming group. On the other hand, general cognitive-motor interventions seemingly have a positive impact on attention, mental flexibility and working memory abilities. Nonetheless these results could not be generalised and were influenced by many methodological factors.

\section{Type of intervention}

An inter-study comparison between technology-based exercise forms and general dual-task methods was not possible. Types of training methods varied from "off-theshelf" computerised games to specifically developed modules. For example, five out of fifteen studies used the interactive step-based game "Dance Dance Revolution" (StepMania) out of which three were SDT intervention types. While Schoene and colleagues [31, 40] implemented a home-based setting, the remaining trials chose a supervised laboratory setting. Three other studies used commercially available games for Nintendo Wii or Kinect Xbox while using various equipment, e.g. an additional balance board. Although the use of modern 
technologies evolved throughout the last decade, a general implementation and usage of exergames still seems unusual in older people. Therefore, the acceptance and feasibility of emerging exergaming modules should be questioned. However, the current findings support the potential of serious gaming and engaging, active and home-based exercise methods for older people $[14,56,57]$.

\section{Dose of effective interventions}

We hypothesised that a higher total intervention dosage would lead to a larger effect size of the intervention. Intervention duration varied between the studies; some trials had a total duration of 6 weeks, while others provided more than 25 weeks of intervention. Trials with a longer total intervention duration and a higher frequency of training sessions seemed to have greater effect size [15, 26-28, 33, 35, 38, 47]. Nevertheless, irrespective of total exercise dose, 24 out of 25 studies showed positive effects on cognitive functions with a training duration of eight to $104 \mathrm{~h}$ (cf. Table 1). The frequency differed from one up to three sessions per week, however, the studies with one session per week had a training duration of at least $60 \mathrm{~min}$ per session. Based on these findings (cf. Tables 1, 3 and 4), we suggest that a total exercise dose of at least $12 \mathrm{~h}$ should be recommended to gain positive effects on EF. Moreover, the duration of all sessions provided per week should be at least $60 \mathrm{~min}$. It has to be noted, that the studies that were not able to improve cognition used the same amount of exercises as shown to be effective to gain positive effects (except of [37] who did not provide information about duration and frequency). Therefore, other reasons such as training specificity or the individual response to cognitive-motor training might have to be considered to explain the results.

\section{Specificity of effective interventions}

Our hypothesis that the training intervention should be task-specific to gain highest benefits on EF was supported by the available study data. The majority of studies that included task-specific training and testing of EF improved the specific domain of EF. As the meta-analyses revealed inconsistent findings for the different dimensions of $\mathrm{EF}$, further research should assess the effects of different complexities of cognitive tasks on specific executive functions. Several reviews have shown effects of acute and regular exercise on cognition [58, 59]. However, a wide interindividual variability has been found in the effects of acute and/or regular exercise [60], suggesting there might be responders and non-responders. Therefore, future studies should address aspects of, e.g. responder analysis [61], dose-response relationships [62] and more specific tailoring of the dual-task exercise program [63]. Of note, a large number of general training principles (for an overview $\mathrm{cf}$.
Herold et al., 2019 [62]) were not addressed in most studies. There was a lack of detail on addition to training specificity and progression, aspects of variation, overload, reversibility, periodisation, and programming in the provided exercise interventions. These aspects of training methods and mechanisms should be addressed in future clinical trials to allow clinical practice recommendations.

\section{Limitations}

This review has some limitations that need to be addressed. Certain interventions were not included in the review, despite training highly coordinated tasks such as dancing. Following our definition of a DT training, a motor task needed to be performed at the same time of an ongoing observational task, e.g. on a screen. We acknowledge that these types of interventions might add further insights when compared to the more specified DT programs. Second, in order not to unduly exclude studies including people with mild cognitive impairment, we used a slightly lower cut-point (MMSE <23) to allow subgroup analyses in cognitively healthy versus people with mild cognitive impairment. These different interpretations of non-cognitively impaired older people might have an impact on the study results. Nevertheless, we did not find a systematic direction, if the training interventions gain higher or lower benefits for participants with lower scores in the MMSE.

\section{Conclusion}

Conventional cognitive-motor interventions as well as technology-based exergames can show positive effects on global cognition and inhibition in healthy older people aged 60 and older. The cognitive domains that were influenced by dual-task training programs varied from attentional skills to inhibitory control or mental set-shifting abilities and the reduction of dual-task costs. New technologies can complement home-based and unsupervised training methods, as a useful tool for older people to independently perform adequate training sessions. Of note, results of the meta-analysis were heterogeneous and need to be interpreted carefully due to differences in interventions, measurements and results. Additionally, the influence of different levels of cognitive demands and the influence on specific executive functions should be investigated. While we recommend a minimal dose of $12 \mathrm{~h}$ with $60 \mathrm{~min}$ per week to achieve effects, this is still preliminary and warrants further research.

\section{Appendix}

Search Strategy:

1 Aged or elder* or senior* or geriatric* or older or aged 80 ) and over $\mathrm{mp}=$ title, abstract, heading word, 
drug trade name, original title, device manufacturer, drug manufacturer, device trade name, keyword, floating subheading word, candidate term word]

2 Dual-task" or exergam* or motor-cognitive or motor\$ cogn" or video gam* or computer\$ gam*$\left.^{*}\right) \cdot \mathrm{mp}$. [mp = title, abstract, heading word, drug trade name, original title, device manufacturer, drug manufacturer, device trade name, keyword, floating subheading word, candidate term word]

3 Intervention or program* or training* or exercise or exercise therapy or physical train*).mp. [mp = title, abstract, heading word, drug trade name, original title, device manufacturer, drug manufacturer, device trade name, keyword, floating subheading word, candidate term word]

4 Cognition or cognitive function* or executive function* or attention*).mp. [mp = title, abstract, heading word, drug trade name, original title, device manufacturer, drug manufacturer, device trade name, keyword, floating subheading word, candidate term word]

\section{Acknowledgements}

KvS is supported by a Human Frontier Science Program Fellowship (LT001080/2017). ML and KD are supported by the Australian National Health and Medical Research Council.

\section{Authors' contributions}

AW, BW, KVS, ML and KD conceived and designed this study and developed the search strategy. AW and BW conducted the search as well as the study selection and quality scoring individually according to the PRISMA statement, while KD, KvS, ML critically reviewed the results. BW performed the meta-analysis for the review. The final manuscript was drafted together by BW and AW and was critically reviewed by KD, KvS and ML. The author(s) read and approved the final manuscript.

\section{Funding}

There was no funding received for this study.

\section{Availability of data and materials}

The supporting data is available via the corresponding author.

\section{Ethics approval and consent to participate}

There was no Ethical approval required as the study was a systematic review. This review was registered at Prospero with the ID: CRD 4208111083.

\section{Consent for publication}

All authors have approved the manuscript and agree with its submission to "European Review of Aging and Physical activity".

\section{Competing interests}

The authors declare that they have no competing interests.

\section{Author details}

'Department of Human Movement Science, University of Hamburg, Hamburg, Germany. ${ }^{2}$ Biological Psychology and Neuroergonomics, Technical University of Berlin, Fasanenstr. 1, D-10623 Berlin, Germany. ${ }^{3}$ Neuroscience Research Australia, Sydney, New South Wales, Australia. ${ }^{4}$ School of Public Health and Community Medicine, Faculty of Medicine, University of New South Wales, Sydney, New South Wales, Australia.
Received: 23 January 2020 Accepted: 13 May 2020

Published online: 02 July 2020

\section{References}

1. Seidler RD, Bernard JA, Burutolu TB, et al. Motor control and aging: links to agerelated brain structural, functional, and biochemical effects. Neurosci Biobehav Rev. 2010;34(5):721-33. https://doi.org/10.1016/j.neubiorev.2009.10.005.

2. Faulkner KA, Redfern MS, Cauley JA, et al. Multitasking: association between poorer performance and a history of recurrent falls. J Am Geriatr Soc. 2007; 55(4):570-6. https://doi.org/10.1111/j.1532-5415.2007.01147.x.

3. Lacour M, Bernard-Demanze L, Dumitrescu M. Posture control, aging, and attention resources: models and posture-analysis methods. Neurophysiol Clin. 2008:38(6):411-21.

4. Wollesen B, Voelcker-Rehage C, Regenbrecht T, Mattes K. Influence of a visual-verbal Stroop test on standing and walking performance of older adults. Neuroscience. 2016;318:166-77.

5. Agmon M, Perry C, Phelan E, Demiris G, Nguyen H. A pilot study of wii fit exergames to improve balance in older adults. J Geriatr Phys Ther (2001). 2011:34(4):161-7.

6. Sherrington C, Whitney JC, Lord SR, Herbert RD, Cumming RG, Close JCT. Effective exercise for the prevention of falls: a systematic review and metaanalysis. J Am Geriatr Soc. 2008;56(12):2234-43. https://doi.org/10.1111/j. 1532-5415.2008.02014.x.

7. Verhaeghen P, Cerella J. Aging, executive control, and attention: a review of meta-analyses. Neurosci Biobehav Rev. 2002;26(7):849-57.

8. Wollesen, Voelcker-Rehage C. Training effects on motor-cognitive dual-task performance in older adults. Eur Rev Aging Phys Act. 2014;1 1(1):5-24. https://doi.org/10.1007/s11556-013-0122-z.

9. Yogev-Seligmann G, Hausdorff JM, Giladi N. The role of executive function and attention in gait. Mov Disord. 2008;23(3):329-42.

10. Diamond A. Executive functions. Annu Rev Psychol. 2013;64:135-68.

11. Hausdorff JM, Yogev G, Springer S, Simon ES, Giladi N. Walking is more like catching than tapping: gait in the elderly as a complex cognitive task. Exp Brain Res. 2005;164(4):541-8.

12. Holtzer R, Verghese J, Xue X, Lipton RB. Cognitive processes related to gait velocity: results from the einstein aging study. Neuropsychology. 2006;20(2):215.

13. Sheridan PL, Solomont J, Kowall N, Hausdorff JM. Influence of executive function on locomotor function: divided attention increases gait variability in alzheimer's disease. J Am Geriatr Soc. 2003:51(11):1633-7.

14. Stanmore E, Stubbs B, Vancampfort D, de Bruin ED, Firth J. The effect of active video games on cognitive functioning in clinical and non-clinical populations: a meta-analysis of randomized controlled trials. Neurosci Biobehav Rev. 2017;78:34-43.

15. Falbo S, Condello G, Capranica L, Forte R, Pesce C. Effects of physicalcognitive dual task training on executive function and gait performance in older adults: a randomized controlled trial. Biomed Res Int. 2016; 2016(5812092):5812092. https://doi.org/10.1155/2016/5812092.

16. Colcombe S, Kramer AF. Fitness effects on the cognitive function of older adults: a meta-analytic study. Psychol Sci. 2003;14(2):125-30.

17. Schaefer $S$, Schumacher $V$. The interplay between cognitive and motor functioning in healthy older adults: findings from dual-task studies and suggestions for intervention. Gerontology. 2011;57(3):239-46. https://doi. org/10.1159/000322197.

18. Tait JL, Duckham RL, Milte CM, Main LC, Daly RM. Influence of sequential vs. simultaneous dual-task exercise training on cognitive function in older adults. Front Aging Neurosci. 2017;9(368):368. https:/doi.org/10.3389/fnagi.2017.00368.

19. Anderson-Hanley C, Maloney M, Barcelos N, Striegnitz K, Kramer A. Neuropsychological benefits of neuro-exergaming for older adults: a pilot study of an interactive physical and cognitive exercise system (ipaces). J Aging Phys Act. 2017;25(1):73-83. https://doi.org/10.1123/japa.2015-0261.

20. Bherer L, Erickson Kl, Liu-Ambrose T. A review of the effects of physical activity and exercise on cognitive and brain functions in older adults. J Aging Res. 2013;2013.

21. Kramer AF, Bherer L, Colcombe SJ, Dong W, Greenough WT. Environmental influences on cognitive and brain plasticity during aging. J Gerontol Ser A Biol Med Sci. 2004:59(9):M940-57.

22. Park DC, Bischof GN. The aging mind: neuroplasticity in response to cognitive training. Dialogues Clin Neurosci. 2013;15(1):109.

23. Moher D, Liberati A, Tetzlaff J, Altman DG. Preferred reporting items for systematic reviews and meta-analyses: the prisma statement. Ann Intern Med. 2009;151(4):264-9. 
24. Maher CG, Sherrington C, Herbert RD, Moseley AM, Elkins M. Reliability of the PEDro scale for rating quality of randomized controlled trials. Phys Ther. 2003;83(8):713-21.

25. Ansai JH, de Andrade LP, de Souza Buto MS, de Vassimon Barroso V, Farche AC, Rossi PG, de Medeiros Takahashi AC. Effects of the addition of a dual task to a supervised physical exercise program on older adults' cognitive performance. J Aging Phys Act. 2017;25(2):234-9. https://doi.org/10.1123/ japa.2016-0094.

26. Azadian E, Torbati HR, Kakhki AR, Farahpour N. The effect of dual task and executive training on pattern of gait in older adults with balance impairment: a randomized controlled trial. Arch Gerontol Geriatr. 2016;62: 83-9. https://doi.org/10.1016/j.archger.2015.10.001

27. Eggenberger $P$, Schumacher $V$, Angst $M$, Theill $N$, de Bruin ED. Does multicomponent physical exercise with simultaneous cognitive training boost cognitive performance in older adults? A 6-month randomized controlled trial with a 1-year follow-up. Clin Interv Aging. 2015;10:1335-49.

28. Kayama H, Okamoto K, Nishiguchi S, Yamada M, Kuroda T, Aoyama T. Effect of a kinect-based exercise game on improving executive cognitive performance in community-dwelling elderly: case control study. J Med Internet Res. 2014;16(2):e61. https://doi.org/10.2196/jmir.3108.

29. Maillot P, Perrot A, Hartley A. Effects of interactive physical-activity videogame training on physical and cognitive function in older adults. Psychol Aging. 2012;27(3):589-600. https://doi.org/10.1037/a0026268.

30. Ordnung $M$, Hoff $M$, Kaminski $E$, Villringer A, Ragert $P$. No overt effects of a 6-week exergame training on sensorimotor and cognitive function in older adults. A preliminary investigation. Front Hum Neurosci. 2017;11(160):160. https://doi.org/10.3389/fnhum.2017.00160

31. Schoene D, Lord SR, Delbaere K, Severino C, Davies TA, Smith ST. A randomized controlled pilot study of home-based step training in older people using videogame technology. PLoS One. 2013;8(3):e57734. https:// doi.org/10.1371/journal.pone.0057734.

32. Bacha JMR, Gomes GCV, de Freitas TB, Viveiro LAP, da Silva KG, Bueno GC, et al. Effects of kinect adventures games versus conventional physical therapy on postural control in elderly people: a randomized controlled trial. Games Health J. 2018;7(1):24-36. https://doi.org/10.1089/g4h.2017.0065.

33. Eggenberger $P$, Wolf $M$, Schumann $M$, de Bruin ED. Exergame and balance training modulate prefrontal brain activity during walking and enhance executive function in older adults. Front Aging Neurosci. 2016;8:66. https:// doi.org/10.3389/fnagi.2016.00066.

34. Hars M, Herrmann FR, Gold G, Rizzoli R, Trombetti A. Effect of music-based multitask training on cognition and mood in older adults. Age Ageing. 2014:43(2):196-200. https://doi.org/10.1093/ageing/aft163.

35. Hiyamizu M, Morioka S, Shomoto K, Shimada T. Effects of dual task balance training on dual task performance in elderly people: a randomized controlled trial. Clin Rehabil. 2012;26(1):58-67.

36. Kitazawa K, Showa S, Hiraoka A, Fushiki Y, Sakauchi H, Mori M. Effect of a dualtask net-step exercise on cognitive and gait function in older adults. J Geriatr Phys Ther. 2015;38(3):133-40. https://doi.org/10.1519/JPT.0000000000000029.

37. Maclean $L M$, Brown $\sqcup$, Astell AJ. The effect of rhythmic musical training on healthy older adults' gait and cognitive function. Gerontologist. 2014;54(4): 624-33. https://doi.org/10.1093/geront/gnt050.

38. Nishiguchi S, Yamada M, Tanigawa T, Sekiyama K, Kawagoe T, Suzuki M, et al. A 12-week physical and cognitive exercise program can improve cognitive function and neural efficiency in community-dwelling older adults: a randomized controlled trial. J Am Geriatr Soc. 2015;63(7):1355-63. https://doi.org/10.1111/jgs.13481

39. Schaettin A, Arner R, Gennaro F, de Bruin ED. Adaptations of prefrontal brain activity, executive functions, and gait in healthy elderly following exergame and balance training: a randomized-controlled study. Front Aging Neurosci. 2016;8(278):278. https://doi.org/10.3389/fnagi.2016.00278.

40. Schoene D, Valenzuela T, Toson B, Delbaere K, Severino C, Garcia J, et al. Interactive cognitive-motor step training improves cognitive risk factors of falling in older adults - a randomized controlled trial. PLoS One. 2015;10(12): e0145161. https://doi.org/10.1371/journal.pone.0145161.

41. Wollesen B, Schulz S, Seydell L, Delbaere K. Does dual task training improve walking performance of older adults with concern of falling? BMC Geriatr. 2017a;17(1):213.

42. Wollesen B, Mattes K, Schulz S, Bischoff LL, Seydell L, Bell JW, von Duvillard SP. Effects of dual-task management and resistance training on gait performance in older individuals: a randomized controlled trial. Front Aging Neurosci. 2017b;9:415.
43. You JH, Shetty A, Jones T, Shields K, Belay Y, Brown D. Effects of dual-task cognitive-gait intervention on memory and gait dynamics in older adults with a history of falls: a preliminary investigation. NeuroRehabilitation. 2009; 24(2):193-8. https://doi.org/10.3233/NRE-2009-0468.

44. Bisson E, Contant B, Sveistrup H, Lajoie Y. Functional balance and dual-task reaction times in older adults are improved by virtual reality and biofeedback training. CyberPsychol Behav. 2007;10(1):16-23.

45. Chuang LY, Hung HY, Huang CJ, Chang YK, Hung TM. A 3-month intervention of dance dance revolution improves interference control in elderly females: a preliminary investigation. Exp Brain Res. 2015;233(4):11818. https://doi.org/10.1007/s00221-015-4196-x.

46. Heiden E, Lajoie Y. Games-based biofeedback training and the attentional demands of balance in older adults. Aging Clin Exp Res. 2010;22(5-6):36773. https://doi.org/10.3275/6733.

47. Morita E, Yokoyama H, Imai D, Takeda R, Ota A, Kawai E, et al. Effects of 2year cognitive(-)motor dual-task training on cognitive function and motor ability in healthy elderly people: a pilot study. Brain Sci. 2018;8(5). https:// doi.org/10.3390/brainsci8050086.

48. Theill N, Schumacher $V$, Adelsberger R, Martin M, Jancke L. Effects of simultaneously performed cognitive and physical training in older adults. BMC Neurosci. 2013;14(103):103. https://doi.org/10.1186/1471-2202-14-103.

49. Netz $Y$, Dwolatzky T, Zinker $Y$, Argov E, Agmon R. Aerobic fitness and multidomain cognitive function in advanced age. Int Psychogeriatr. 2011;23(1):114-24.

50. Aquirre-Acevedo DC, Jaimes-Barragán F, Henao E, Tirado V, Muñoz C, Reiman EM, et al. Diagnostic accuracy of CERAD total score in a Colombian cohort with mild cognitive impairment and Alzheimer's disease affected by E280A mutation on presenilin-1 gene. Int Psychogeriatr. 2016;28(3):503-10.

51. Andrés P, Guerrini C, Phillips LH, Perfect TJ. Differential effects of aging on executive and automatic inhibition. Dev Neuropsychol. 2008;33(2): 101-23.

52. Desjardins-Crepeau L, Berryman N, Fraser SA, Vu TT, Kergoat MJ, Li KZ, et al. Effects of combined physical and cognitive training on fitness and neuropsychological outcomes in healthy older adults. Clin Interv Aging. 2016;11:1287-99. https://doi.org/10.2147/Cia.S115711.

53. Gregory MA, Boa Sorte Silva NC, Gill DP, McGowan CL, Liu-Ambrose T, Shoemaker JK, et al. Combined dual-task gait training and aerobic exercise to improve cognition, mobility, and vascular health in community-dwelling older adults at risk for future cognitive decline $\langle$ sup $\rangle 1</$ sup $\rangle$. J Alzheimers Dis. 2017;57(3):747-63.

54. Künstler E, Finke K, Günther A, Klingner C, Witte O, Bublak P. Motor-cognitive dual-task performance: effects of a concurrent motor task on distinct components of visual processing capacity. Psychol Res. 2018;82(1):177-85.

55. Kelly ME, Loughrey D, Lawlor BA, Robertson IH, Walsh C, Brennan S. The impact of exercise on the cognitive functioning of healthy older adults: a systematic review and meta-analysis. Ageing Res Rev. 2014;16:12-31.

56. Chi H, Agama E, Prodanoff ZG. Developing serious games to promote cognitive abilities for the elderly. In: Paper presented at the 2017 IEEE 5th international conference on serious games and applications for health (SeGAH); 2017.

57. Zhang F, Kaufman D. Physical and cognitive impacts of digital games on older adults: a meta-analytic review. J Appl Gerontol. 2016;35(11):1189-210. https://doi.org/10.1177/0733464814566678.

58. Erickson Kl, Kramer AF. Aerobic exercise effects on cognitive and neural plasticity in older adults. Br J Sports Med. 2009;43(1):22-4.

59. Bherer L. Cognitive plasticity in older adults: effects of cognitive training and physical exercise. Ann N Y Acad Sci. 2015;1337(1):1-6.

60. Schwarck S, Schmicker M, Dordevic M, Rehfeld K, Müller N, Müller P. Interindividual differences in cognitive response to a single bout of physical exercise-a randomized controlled cross-over study. J Clin Med. 2019;8(8):1101.

61. Dankel SJ, Kang M, Abe T, Loenneke JP. Resistance training induced changes in strength and specific force at the fiber and whole muscle level: a meta-analysis. Eur J Appl Physiol. 2019;119(1):265-78.

62. Herold F, Müller P, Gronwald T, Müller NG. Dose-response matters!-a perspective on the exercise prescription in exercise-cognition research. Front Psychol. 2019;10.

63. Müllers P, Taubert M, Müller NG. Physical exercise as personalized medicine for dementia prevention? Front Physiol. 2019;10.

\section{Publisher's Note}

Springer Nature remains neutral with regard to jurisdictional claims in published maps and institutional affiliations. 